Publ. Mat. 61 (2017), 445-474

DOI: 10.5565 /PUBLMAT6121705

\title{
EXAMPLES OF FINITE-DIMENSIONAL HOPF ALGEBRAS WITH THE DUAL CHEVALLEY PROPERTY
}

\author{
Nicolás Andruskiewitsch, CÉsar Galindo, and \\ MONIQUe MÜLLER
}

\begin{abstract}
We present new Hopf algebras with the dual Chevalley property by determining all semisimple Hopf algebras Morita-equivalent to a group algebra over a finite group, for a list of groups supporting a non-trivial finite-dimensional Nichols algebra.
\end{abstract}

2010 Mathematics Subject Classification: 16 T05.

Key words: Hopf algebras, fusion categories, pointed Hopf algebras, braided tensor categories.

\section{Introduction}

1.1. A Hopf algebra has the dual Chevalley property if the tensor product of two simple comodules is semisimple, or equivalently if its coradical is a (cosemisimple) Hopf subalgebra. These Hopf algebras are interesting by various reasons, among them the Lifting Method for their classification $[\mathbf{A S}]$. Particular classes are the pointed (the coradical is a group algebra) and copointed (the coradical is the algebra of functions on a finite or reductive group) Hopf algebras. However few examples out of these classes has been discussed in the literature [CDMM, Mom]. The purpose of this paper is to present explicit examples of Hopf algebras with the dual Chevalley property.

1.2. In this paper the underlying field $\mathbb{k}$ is algebraically closed of characteristic 0 . Then the coradical of a finite-dimensional Hopf algebra with

N. Andruskiewitsch and M. Müller were partially supported by ANPCyT-Foncyt, CONICET, and Secyt (UNC). C. Galindo was partially supported by the FAPA funds from Vicerrectoría de Investigaciones de la Universidad de los Andes. Part of this work was done during visits of N. Andruskiewitsch to the Universidad de los Andes. 
the dual Chevalley property is a semisimple Hopf algebra. Let us consider the problem of constructing (and eventually classifying) finite-dimensional Hopf algebras $A$ whose coradical $A_{0}$ is isomorphic to a fixed semisimple Hopf algebra $H$. For this, we need to address three problems:

(a) To find (classify) the Yetter-Drinfeld modules $V \in{ }_{H}^{H} \mathcal{Y D}$ such that the dimension of the Nichols algebra $\mathfrak{B}(V)$ is finite. Then $\mathcal{A}(V)=$ $\mathfrak{B}(V) \# H$ is a finite-dimensional Hopf algebra with $\mathcal{A}(V)_{0} \simeq H$.

(b) To find (classify) the deformations, or liftings, of $\mathcal{A}(V)$; that is, Hopf algebras $A$ such that gr $A$ (the graded Hopf algebra with respect the coradical filtration) is isomorphic to $\mathcal{A}(V)$.

(c) If $A$ is a finite-dimensional Hopf algebra with $A_{0} \simeq H$, then prove that there exists $V$ such that gr $A \simeq \mathcal{A}(V)$.

1.3. Two fusion categories $\mathcal{C}$ and $\mathcal{D}$ are Morita-equivalent if there exists an indecomposable $\mathcal{C}$-module category $\mathcal{M}$ such that $\mathcal{D}$ is tensor equivalent to $\operatorname{End}_{\mathcal{C}}(\mathcal{M})[\mathbf{M u ̈}]$; equivalently, if their centers are equivalent as braided tensor categories [ENO, Theorem 3.1]. In this case we write $\mathcal{C} \sim$ Mor $\mathcal{D}$.

Two semisimple Hopf algebras $K$ and $H$ are Morita-equivalent (denoted by $K \sim_{\text {Mor }} H$ ) iff $\operatorname{Rep} K \sim_{\text {Mor }} \operatorname{Rep} H$, iff ${ }_{K}^{K} \mathcal{Y} \mathcal{D}$ and ${ }_{H}^{H} \mathcal{Y D}$ are equivalent as braided tensor categories. (This is not the same as being Morita-equivalent as algebras.) When this is the case, the braided equivalence $\mathcal{F}:{ }_{K}^{K} \mathcal{Y D} \rightarrow{ }_{H}^{H} \mathcal{Y} \mathcal{D}$ preserves Nichols algebras, i.e. $\mathcal{F}(\mathfrak{B}(V)) \simeq$ $\mathfrak{B}(\mathcal{F}(V))$. In consequence, if Problem (a) above is solved for $K$, then so is for $H$. Also, Problem (c) is equivalent to: if $\mathfrak{B} \rightarrow \mathfrak{B}(V)$ is a finitedimensional pre-Nichols algebra in ${ }_{K}^{K} \mathcal{Y} \mathcal{D}$, then necessarily $\mathfrak{B} \simeq \mathfrak{B}(V)$. Therefore, if Problem (c) above is solved for $K$, then so is for $H$.

In this paper we construct Hopf algebras with the Chevalley property over a semisimple Hopf algebra $H$ that is Morita-equivalent to a group algebra $K=\mathbb{k} G, G$ a finite group, provided we know examples, or even better the classification, of $V \in{ }_{K}^{K} \mathcal{Y} \mathcal{D}$ with $\operatorname{dim} \mathfrak{B}(V)<\infty$.

1.4. Let $G$ be a finite group. The characterization of all semisimple Hopf algebras Morita-equivalent to $\mathbb{k} G$ follows from [Os] as we briefly recall now.

○ Duals. If $H$ is a semisimple Hopf algebra, then $H^{*} \sim_{\text {Mor }} H$ - just take $\mathcal{M}=\operatorname{vec}_{\mathbb{k}}$. In particular $\operatorname{vec}_{G} \sim_{\text {Mor }} \operatorname{Rep} \mathbb{k} G$, where $\operatorname{vec}_{G}$ is the category of finite-dimensional $G$-graded vector spaces.

- Group-theoretical Hopf algebras. Let $F, \Gamma<G$ be such that $G=F \Gamma-$ but $F \cap \Gamma$ may be nontrivial. Given a suitable pair $(\alpha, \beta) \in H^{2}\left(F, \mathbb{k}^{\times}\right) \times$ $H^{2}\left(\Gamma, \mathbb{k}^{\times}\right)$, cf. Definition 2.1, there is a corresponding Hopf algebra 
$H_{\alpha \beta}^{G}(F, \Gamma)$ such that $H_{\alpha \beta}^{G}(F, \Gamma) \sim_{\text {Mor }} \mathbb{k} G$. The collection $(F, \alpha, \Gamma, \beta)$ is called a group-theoretical datum for $G$. These are all Hopf algebras arising from fiber functors of all fusion categories Morita-equivalent to $\operatorname{vec}_{G}[\mathbf{O s}]$, therefore all $H \sim_{\text {Mor }} \mathbb{k} G$ are like this. See Subsection 2.2 for more details. Notice however that to decide when two of these Hopf algebras are isomorphic is not evident.

We describe next some particular instances of this notion.

- Twistings. Two finite-dimensional Hopf algebras $H$ and $H^{\prime}$ are twistequivalent if and only if $\operatorname{Rep} H$ and $\operatorname{Rep} H^{\prime}$ are tensor equivalent [EG2, Sc1]. Therefore, if $J \in \mathbb{k} G \otimes \mathbb{k} G$ is a twist, then $(\mathbb{k} G)^{J} \sim_{\text {Mor }} \mathbb{k} G$. Furthermore, if $U$ is a pointed Hopf algebra with $G(U) \simeq G$, then $J \in$ $U \otimes U$ is a twist and the Hopf algebra $U^{J}$, with coradical $(\mathbb{k} G)^{J}$, has the dual Chevalley property. Hence, if Problem (b) is solved for $\mathbb{k} G$, then so is for $(\mathbb{k} G)^{J}$.

- Abelian extensions $[\mathbf{K a}, \mathbf{T a}]$. Assume that $G=F \Gamma$ is an exact factorization (i.e., $F, \Gamma<G$ with $G=F \Gamma, F \cap \Gamma=1$ ) and that $H$ fits into an exact sequence

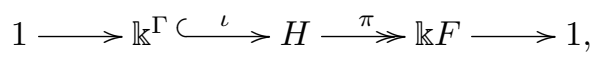

associated to this factorization. Let $(\sigma, \tau) \in \operatorname{Opext}\left(\mathbb{k}^{\Gamma}, \mathbb{k} F\right)$ be the corresponding pair of 2-cocycles. Thus $H=\mathbb{k}^{\Gamma \tau} \#_{\sigma} \mathbb{k} F$ is a bicrossed product. Let $\omega \in Z^{3}\left(G, \mathbb{k}^{\times}\right)$be the 3 -cocycle arising from $(\sigma, \tau)$ in the Kac exact sequence; we say that $H$ has 3 -cocycle $\omega$. Albeit $\operatorname{Rep} \mathbb{k} G$ and $\operatorname{Rep} H$ are not necessarily tensor-equivalent, $D(H)$ is isomorphic to a twist of $D^{\omega}(\mathbb{k} G)$ [Nat2, Theorem 1.3]. Hence, if $\omega=1$, then $H \sim_{\text {Mor }} \mathbb{k} G$ by [ENO, Theorem 3.1].

1.5. As is well-known, the Nichols algebra $\mathfrak{B}(V)$ depends essentially only on the underlying braided vector space to the Yetter-Drinfeld module $V$. Here we shall not consider braided vector spaces of diagonal type - except for Yetter-Drinfeld modules over some dihedral groups, see Table 8 in Section 9. We focus on braided vector spaces of rack type, see e.g. [AG1] or $[\mathbf{A F G V}]$. For more examples with braided vector spaces of diagonal type see [CDMM, Mom]. Let $(X, \mathbf{q})$ be a pair where $X$ is a rack and $q$ a 2-cocycle, let $V$ be the associated braided vector space and assume that $\mathfrak{B}(V)$ is finite-dimensional, cf. [HLV]. We consider a group $G$ such that $V$ is realized in ${ }_{\mathbb{k} G}^{\mathbb{k} G} \mathcal{Y} \mathcal{D}$. We then compute all group-theoretical data $(F, \alpha, \Gamma, \beta)$ for $G$. Consequently, $H=H_{\alpha \beta}^{G}(F, \Gamma) \sim_{\text {Mor }} \mathbb{k} G$ and there is $V^{\prime} \in{ }_{H}^{H} \mathcal{Y D}$ such that $\mathfrak{B}\left(V^{\prime}\right) \simeq \mathfrak{B}(V)$, as algebras and coalgebras. We summarize our computations in Table 1. 


\begin{tabular}{|c|c|c|c|c|}
\hline$(X, \mathbf{q})$ & $\operatorname{dim} \mathfrak{B}(V)$ & Reference & $G$ & $H \sim_{\text {Mor }} \mathbb{k} G$ \\
\hline $\mathcal{D}_{3},-1$ & 12 & {$[\mathbf{M S}]$} & $C_{3} \rtimes C_{6}$ & Prop. 3.1 \\
\hline$\left(\mathrm{Q}_{5,2},-1\right),\left(\mathrm{Q}_{5,3},-1\right)$ & 1280 & {$[\mathbf{A G 1}]$} & $C_{5} \rtimes_{2} C_{20}$ & Table 3 \\
\hline$\left(\mathcal{O}_{2}^{4},-1\right),\left(\mathcal{O}_{2}^{4}, \chi\right),\left(\mathcal{O}_{4}^{4},-1\right)$ & 576 & {$[\mathbf{F K}, \mathbf{M S}]$} & $\mathbb{S}_{4}$ & Table 4 \\
\hline$\left(\mathcal{O}_{2}^{5},-1\right),\left(\mathcal{O}_{2}^{5}, \chi\right)$ & 8294400 & {$[\mathbf{F K}, \mathbf{G r 2}, \mathbf{G G}]$} & $\mathbb{S}_{5}$ & Table 5 \\
\hline$(\mathcal{T},-1)$ & 72 & {$[\mathbf{G r 1}]$} & $\mathbb{A}_{4} \times C_{2}$ & Table 6 \\
\hline$\left(\mathrm{Q}_{7,3},-1\right),\left(\mathrm{Q}_{7,5},-1\right)$ & 326592 & {$[\mathbf{G r 2}]$} & $C_{7} \rtimes_{3} C_{6}$ & Prop. 8.1 \\
\hline
\end{tabular}

TABLE 1.

We describe new examples in Theorems 3.2, 4.3, 5.3, 6.2, 7.3, 8.2, and 9.5 .

Remark 1.1. (i) There are groups $G$ that admit a finite-dimensional Nichols algebra but no non-trivial $H$ with $H \sim_{\text {Mor }} \mathbb{k} G$. For instance $\left(\mathcal{D}_{3},-1\right)$ corresponds to some $V \in \mathbb{k S}_{\mathbb{R S}_{3}}^{\mathbb{R}_{3}} \mathcal{Y D}$ but $H \sim_{\text {Mor }} \mathbb{k} \mathbb{S}_{3}$ implies $H \simeq$

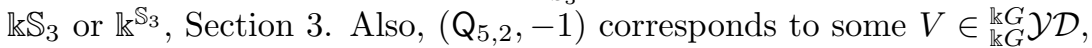
where $G=\mathbb{k}\left(C_{5} \rtimes_{2} C_{4}\right)$; but $H \sim_{\text {Mor }} \mathbb{k} G$ implies $H \simeq \mathbb{k} G$ or $\mathbb{k}^{G}$, Section 4.

(ii) Let $G$ be as in Table 1 or a dihedral group $D_{n}$. By Lemma 2.2(ii), there is no group $G^{\prime} \not 4 G$ with $\mathbb{k} G^{\prime} \sim_{\text {Mor }} \mathbb{k} G$.

(iii) If $J \in \mathbb{k} G \otimes \mathbb{k} G$ is a twist and $U$ is a pointed Hopf algebra with $G(U) \simeq G$, then the Hopf algebra $U^{J}$, with coradical $(\mathbb{k} G)^{J}$, has a rather concrete description. Otherwise, if $H \sim_{\text {Mor }} \mathbb{k} G$, then the braided equivalence $\mathcal{F}:{ }_{\mathbb{k} G}^{\mathbb{k} G} \mathcal{Y} \mathcal{D} \rightarrow{ }_{H}^{H} \mathcal{Y} \mathcal{D}$ is not quite explicit. In this way, neither the structure of the Yetter-Drinfeld module $\mathcal{F}(V)$ nor the defining relations of $\mathfrak{B}(\mathcal{F}(V))$ are not known, and the description of the liftings is problematic. Notice that a direct relation between liftings of $\mathfrak{B}(V) \# \mathbb{k} G$ and liftings of $\mathfrak{B}(\mathcal{F}(V)) \# H$ is not expected. For instance, if $G=\mathbb{S}_{3}$ and $\mathfrak{B}(V)$ is the 12 -dimensional Nichols algebra, then $\mathfrak{B}(V) \# \mathbb{k} \mathbb{S}_{3}$ has exactly one non-trivial lifting $[\mathbf{A G 2}]$; whereas $\mathfrak{B}(V) \# \mathbb{K}^{\mathbb{S}_{3}}$ has infinitely many non-isomorphic liftings $[\mathbf{A V}]$.

We use GAP (see $[\mathbf{G A P}]$ ) for some of the computations with finite groups. For most of the computations of finite group cohomology, we use the GAP package HAP and the natural isomorphism $H^{n}\left(-, \mathbb{k}^{\times}\right) \cong$ $H^{n+1}\left({ }_{-}, \mathbb{Z}\right)$.

Acknowledgements. We thank Graham Ellis for helping us to use the GAP package HAP, Leandro Vendramin and Cristian Vay for kindly answering some questions, and Agustín García Iglesias for very useful conversations. 
1.6. Preliminaries and notations. As customary we refer to group algebras and their duals as trivial Hopf algebras. We denote by 1 the identity element of a group. If $n \in \mathbb{N}$, then $C_{n}$ is a cyclic group of order $n$. If $G$ is a group, then the notation $F<G$ means that $F$ is a subgroup of $G$, while $F \triangleleft G$ means that $F<G$ is normal. If $\triangleright$ is an action of a group $G$ on a set $F$, then we denote by $F^{G}$ the subset of $F$ of points fixed by $\triangleright$. The dihedral group of order $2 n$ is denoted by $D_{n}$. We denote multiplicatively the cohomology groups $H^{n}\left(G, \mathbb{k}^{\times}\right)$. Occasionally, we denote by the same letter an element in $H^{n}\left(G, \mathbb{k}^{\times}\right)$and any of its representatives. Let $a, b \in \mathbb{Z}$, the lowest common multiple of $a$ and $b$ is denoted by $[a, b]$ and the greatest common divisor of $a$ and $b$ is denoted by $(a, b)$. We denote by $\mathbb{G}_{n}=\left\{z \in \mathbb{k}^{\times}: z^{n}=1\right\}, n \in \mathbb{N}$, and by $\mathbb{G}_{n}^{\prime}$ the primitive ones.

One says that $\alpha \in Z^{2}\left(G, \mathbb{k}^{\times}\right)$is non-degenerate if and only if the twisted group algebra $\mathbb{k}_{\alpha} G$ is simple; see e.g. [Da, Mov]. Note that this definition only depends on the cohomology class of $\alpha$.

If $A$ is an abelian group and $T \in \operatorname{Aut} A$, then $A$ is a rack (called affine) with $x \triangleright y=(\mathrm{id}-T) x+T y, x, y \in A$. This is denoted by $\mathrm{Q}_{A, T}$; or $\mathrm{Q}_{q, b}$ when $A \simeq \mathbb{F}_{q}$, where $q$ is a prime power, and $T \in$ Aut $\mathbb{F}_{q}$, $T(x)=b x, x \in \mathbb{F}_{q}, b \in \mathbb{F}_{q}^{\times} \simeq C_{q-1}$. Suppose that the order of $b$ divides $q-1$. Then the rack $\mathrm{Q}_{q, b}$ can be realized as a conjugacy class of the group $C_{q} \rtimes_{b} C_{q-1}$, where the subscript $b$ describes the action. Another exception to the notation is $\mathcal{D}_{n}=\mathrm{Q}_{C_{n}, T}$, where $T(x)=-x$, $x \in C_{n}$ (the so-called dihedral rack). Also $\mathcal{T}=\mathrm{Q}_{4, b}, b \in \mathbb{F}_{4}$ irreducible, is called the tetrahedral rack. Meanwhile, $\mathcal{O}_{j}^{n}$ is the conjugacy class of $j$-cycles in $\mathbb{S}_{n}$.

\section{Semisimple Hopf algebras}

Let $G$ be a finite group.

2.1. Twists. Twists in $\mathbb{k} G$ are classified, up to gauge equivalence, by conjugacy classes of pairs $(S, \alpha)$, where $S<G$ and $\alpha \in H^{2}\left(S, \mathbb{k}^{\times}\right)$is non-degenerate [Mov, EG1]. Hence $S$ is solvable and $|S|$ is a square.

2.2. Group-theoretical Hopf algebras. Let $\omega \in Z^{3}\left(G, \mathbb{k}^{\times}\right)$. The tensor category of finite-dimensional $G$-graded vector spaces with associativity constraint given by $\omega$ is denoted by $\operatorname{vec}_{G}^{\omega}$. The tensor categories $\operatorname{vec}_{G}^{\omega}$ and $\operatorname{vec}_{G}^{\nu}$ are tensor equivalent iff $f^{*}(\bar{\omega})=\bar{\nu}$ in $H^{3}\left(G, \mathbb{k}^{\times}\right)$for some $f \in$ Aut $G$. If $F<G$ and $\alpha \in C^{2}\left(F, \mathbb{k}^{\times}\right)$such that $d \alpha=\omega_{\mid F \times F \times F}$, then the twisted group algebra $\mathbb{k}_{\alpha} F$ is associative in $\operatorname{vec}_{G}^{\omega}$. The category $\mathcal{C}(G, \omega ; F, \alpha)$ of $\mathbb{k}_{\alpha} F$-bimodules in $\operatorname{vec}_{G}^{\omega}$ is a fusion category and 
$\mathcal{C}(G, \omega ; F, \alpha) \sim_{\text {Mor }} \operatorname{vec}_{G}^{\omega}$. By Tannaka duality, to describe the semisimple Hopf algebras $H$ such that Corep $H$ is tensor equivalent to $\mathcal{C}(G, \omega ; F, \alpha)$ is tantamount to describe the fiber functors from $\mathcal{C}(G, \omega ; F, \alpha)$ to $\operatorname{vec}_{\mathbb{k}}$, that is contained in the more general description of indecomposable module categories over $\mathcal{C}(G, \omega ; F, \alpha)$ [Os, Corollary 3.1]. These Hopf algebras are called group-theoretical. By [Sc2], the centers of $\mathcal{C}(G, \omega ; F, \alpha)$ and $\operatorname{vec}_{G}^{\omega}$ are equivalent as braided tensor categories.

We are interested in group-theoretical Hopf algebras $H \sim_{\text {Mor }} \mathbb{k} G$, i.e. with $\omega=1$. Concretely, we introduce the following terminology.

Definition 2.1. A group-theoretical datum for $G$ is a collection $(F, \alpha, \Gamma, \beta)$ where $F, \Gamma<G, \alpha \in H^{2}\left(F, \mathbb{k}^{\times}\right)$, and $\beta \in H^{2}\left(\Gamma, \mathbb{k}^{\times}\right)$, satisfy

- $G=F \Gamma$;

- $\alpha_{\mid F \cap \Gamma} \cdot \beta_{\mid F \cap \Gamma}{ }^{-1}$ is non-degenerate in $F \cap \Gamma$.

Let $(F, \alpha, \Gamma, \beta)$ be a group-theoretical datum for $G$ and choose representatives $\underline{\alpha}, \underline{\beta}$. By $[\mathbf{O s}]$, there is a Hopf algebra $H=H_{\underline{\alpha}}^{G} \underline{\beta}(F, \Gamma)$ with Corep $H$ tensor equivalent to $\mathcal{C}(G, 1 ; F, \alpha)$, and a fortiori $\bar{H} \sim_{\text {Mor }}$ $\mathbb{k} G$; we say that $H$ is a group-theoretical Hopf algebra over $G$. Up to isomorphisms, $H$ only depends on $\alpha$ and $\beta$; so we loosely denote $H=H_{\alpha \beta}^{G}(F, \Gamma)$. Conversely, every Hopf algebra $H$ with $H \sim_{\text {Mor }} \mathbb{k} G$ is isomorphic to $H_{\alpha \beta}^{G}(F, \Gamma)$ for some group-theoretical datum $(F, \alpha, \Gamma, \beta)$ for $G$ by $[\mathbf{O s}]$, as sketched above.

Let $\theta \in$ Aut $G$ and $\gamma \in H^{2}\left(G, \mathbb{k}^{\times}\right)$. If $(F, \alpha, \Gamma, \beta)$ is a group-theoretical datum for $G$, then $\left(\theta(F), \theta_{*}\left(\alpha \gamma_{\mid F}\right), \theta(\Gamma), \theta_{*}\left(\beta \gamma_{\mid \Gamma}\right)\right)$ is again one. Here $\theta_{*}\left(\alpha \gamma_{\mid F}\right)$ is the pushforward cocycle, i.e. $\theta_{*}\left(\alpha \gamma_{\mid F}\right)(\theta(a), \theta(b))=$ $\alpha(a, b) \gamma(a, b)$, for $a, b \in F$ and analogously for $\theta_{*}\left(\beta \gamma_{\mid \Gamma}\right)$. Thus Aut $G \ltimes$ $H^{2}\left(G, \mathbb{k}^{\times}\right)$acts on the set of group-theoretical data; we say that two group-theoretical data are equivalent if they belong to the same orbit of this action.

Lemma 2.2. Let $(F, \alpha, \Gamma, \beta)$ be a group-theoretical datum for $G$.

(i) If $\theta \in$ Aut $G$ and $\gamma \in H^{2}\left(G, \mathbb{k}^{\times}\right)$, then as Hopf algebras

$$
H_{\alpha \beta}^{G}(F, \Gamma) \simeq H_{\theta_{*}\left(\alpha \gamma_{\mid F}\right) \theta_{*}\left(\beta \gamma_{\mid \Gamma}\right)}^{G}(\theta(F), \theta(\Gamma)) .
$$

That is, equivalent group-theoretical data produce isomorphic Hopf algebras.

(ii) $H_{\alpha \beta}^{G}(F, \Gamma)$ is cocommutative (resp. commutative) iff $F \triangleleft G$ is abelian and $\alpha \in H^{2}\left(F, \mathbb{k}^{\times}\right)^{\text {ad } G}$ (resp. $\Gamma \triangleleft G$ is abelian and $\left.\beta \in H^{2}\left(\Gamma, \mathbb{k}^{\times}\right)^{\text {ad } G}\right)$. If $H=H_{\alpha \beta}^{G}(F, \Gamma)$ is cocommutative then $\mathbb{k} G(H) \sim_{\text {Mor }} \mathbb{k} G$ and every $G^{\prime}$ such that $\mathbb{k} G^{\prime} \sim_{\text {Mor }} \mathbb{k} G$ arises in this way. 
(iii) $H_{\alpha \beta}^{G}(F, \Gamma)^{*} \simeq H_{\beta \alpha}^{G}(\Gamma, F)$.

(iv) $H_{\alpha 1}^{G}(F, G)$ is a twisting of $\mathbb{k} G$ and every twisting is of this form.

(v) If $F \cap \Gamma=1$, then $H_{\alpha \beta}^{G}(F, \Gamma)$ is an abelian extension of $\mathbb{k}^{F}$ by $\mathbb{k} \Gamma$ :

$$
\mathbb{k}^{F} \longrightarrow H_{\alpha \beta}^{G}(F, \Gamma) \longrightarrow \mathbb{k} \Gamma \text {. }
$$

Proof: (i) Let $H=H_{\alpha \beta}^{G}(F, \Gamma), \mathcal{C}=\operatorname{vec}_{G}, A=\mathbb{k}_{\alpha} F$, and $B=\mathbb{k}_{\beta} \Gamma$. Clearly $H$ is the Tannakian reconstruction of the fiber functor

$$
{ }_{A} \mathcal{C}_{A} \longrightarrow \operatorname{End}\left({ }_{A} \mathcal{C}_{B}\right) \simeq_{\otimes} \operatorname{vec}_{\mathbb{k}} .
$$

If $(\theta, \gamma) \in \operatorname{Aut} G \ltimes Z^{2}\left(G, \mathbb{k}^{\times}\right)$, then it induces a tensor automorphism $\left(\theta_{*}, \gamma\right): \operatorname{vec}_{G} \rightarrow \operatorname{vec}_{G}$ by

$$
\mathbb{k}_{g} \longmapsto \mathbb{k}_{\theta(g)}, \quad \gamma_{\mathbb{k}_{g}, \mathbb{k}_{h}}:=\gamma(g, h) \operatorname{id}_{\mathbb{k}_{\theta(g h)}}: \mathbb{k}_{\theta(g)} \otimes \mathbb{k}_{\theta(h)} \longrightarrow \mathbb{k}_{\theta(g h)} .
$$

The object $\theta_{*}(A)=\oplus_{g \in F} \mathbb{k}_{\theta(g)}$ is an algebra in $\mathcal{C}$ with multiplication $u_{\theta(g)} u_{\theta(h)}=\gamma(g, h) \theta(g, h) u_{\theta(g h)}$. The tensor automorphism $\left(\theta_{*}, \gamma\right)$ induces a tensor equivalence $\left(\theta_{*}, \gamma\right):{ }_{A} \mathcal{C}_{A} \rightarrow{ }_{\theta_{*}(A)} \mathcal{C}_{\theta_{*}(A)}$ such that the following diagram of tensor functors commutes:

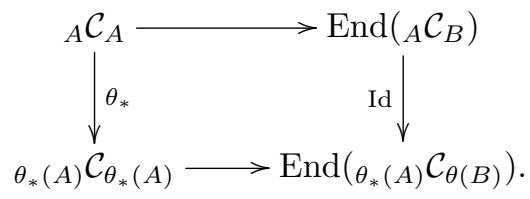

The Tannakian reconstruction of ${ }_{\theta(A)} \mathcal{C}_{\theta(A)} \rightarrow \operatorname{End}\left({ }_{\theta(A)} \mathcal{C}_{\theta(B)}\right)$ is

$$
H_{\theta(F) \theta(\Gamma)}^{G}\left(\theta_{*}\left(\alpha \gamma_{\mid F}\right), \theta_{*}\left(\beta \gamma_{\mid \Gamma}\right),\right.
$$

so by Tannakian formalism and the commutativity of (2.1), the Hopf algebras $H_{\alpha \beta}^{G}(F, \Gamma)$ and $H_{\theta(F) \theta(\Gamma)}^{G}\left(\theta_{*}\left(\alpha \gamma_{\mid F}\right), \theta_{*}\left(\beta \gamma_{\mid \Gamma}\right)\right.$ are isomorphic.

(ii) A semisimple Hopf algebra $H$ is cocommutative, respectively commutative, if and only if Corep $H$, respectively $\operatorname{Rep} H$, is pointed. By [Na, Theorem 3.4], $\mathcal{C}(G, 1 ; F, \alpha)$ is pointed if and only if $F$ is a normal abelian subgroup of $G$ and $\alpha$ is ad $G$-invariant. This implies the claim.

(iii) Let $(F, \alpha, \Gamma, \beta)$ be a group-theoretical datum for $G$. We have associated two fusion categories $\mathbb{k}_{\alpha} F\left(\operatorname{vec}_{G}\right)_{\mathbb{k}_{\alpha} F}, \mathbb{k}_{\beta} \Gamma\left(\operatorname{vec}_{G}\right)_{\mathbb{k}_{\beta} \Gamma}$, and the bimodule category of rank one $\mathbb{k}_{\alpha} F\left(\operatorname{vec}_{G}\right)_{\mathbb{k}_{\beta} \Gamma}$. The Hopf algebra $H_{\alpha \beta}^{G}(F, \Gamma)$ (resp. $H_{\beta \alpha}^{G}(\Gamma, F)$ ) is by definition the Tannaka reconstruction of $\mathbb{k}_{\alpha} F\left(\operatorname{vec}_{G}\right)_{\mathbb{k}_{\alpha} F}$ (resp. $\left.\mathbb{k}_{\beta} \Gamma\left(\operatorname{vec}_{G}\right)_{\mathbb{k}_{\beta} \Gamma}\right)$ respect to the fiber functor defined 
by the left (resp. right) module category $\mathbb{k}_{\alpha} F\left(\operatorname{vec}_{G}\right)_{\mathbb{k}_{\beta} \Gamma}$. Now, it follows by [Ya, Appendix C] that $H_{\alpha \beta}^{G}(F, \Gamma)^{*} \simeq H_{\beta \alpha}^{G}(\Gamma, F)$.

(iv) The fusion category

$$
\operatorname{Rep} H_{\alpha 1}^{G}(F, G) \simeq \operatorname{Corep} H_{1 \alpha}^{G}(G, F) \simeq \mathcal{C}(G, 1 ; G, 1)=_{\mathbb{k} G}\left(\operatorname{vec}_{G}\right)_{\mathbb{k} G}
$$

is tensor equivalent to $\operatorname{Rep} G$. Then $H_{\alpha 1}^{G}(F, G)$ is twist equivalent to $\mathbb{k} G$. Conversely, if $H \simeq(\mathbb{k} G)^{J}$, then $\operatorname{Rep} H \simeq \operatorname{Rep} G \simeq \mathcal{C}(G, 1 ; G, 1)$. Thus $H \simeq H_{\alpha 1}^{G}(F, G)$ for some $F<G$ and $\alpha \in Z^{2}\left(F, \mathbb{C}^{\times}\right)$non-degenerate.

(v) Let $A$ be any extension of $\mathbb{k}^{F}$ by $\mathbb{k} \Gamma$ with 3 -cocycle $\omega$. By [Sc3, Lemma 6.3.1], $A^{J(\beta)}$ and $A_{\alpha}$ are extensions of $\mathbb{k}^{F}$ by $\mathbb{k} \Gamma$ with 3 -cocycle $\omega$. Now by [Nat2, Remark 4.6], $H=H_{\alpha \beta}^{G}(\omega, F, \Gamma) \simeq A_{\alpha}^{J(\beta)}=\left(A^{J(\beta)}\right)_{\alpha}$; notice that $A_{\alpha, \beta}^{G}(\omega, \Gamma, F) \simeq H^{*}$, because of the convention in [Nat2] that $\operatorname{Rep} A_{\alpha, \beta}^{G}(\omega, \Gamma, F)$ is tensor equivalent to $\mathcal{C}(G, \omega ; F, \alpha)$. Hence $H$ is an extension of $\mathbb{k}^{F}$ by $\mathbb{k} \Gamma$ with 3 -cocycle $\omega$, applying twice [Sc3, Lemma 6.3.1]. Since the split extension $\mathbb{k}^{F} \# \mathbb{k} \Gamma$ has 3 -cocycle $1, H_{\alpha \beta}^{G}(F, \Gamma)$ turns out to be an abelian extension.

We shall compute all group-theoretical data of some specific groups and then determine the isomorphism classes of the corresponding grouptheoretical Hopf algebras. We present now some auxiliary results for this end.

Remark 2.3. Let $(F, \alpha, \Gamma, \beta)$ be a group-theoretical datum for $G$. If $\gamma \in H^{2}\left(G, \mathbb{k}^{\times}\right)$, such that $\left.\gamma\right|_{F}=\alpha^{-1}$, then $(F, \alpha, \Gamma, \beta)$ and $\left(F, 1, \Gamma,\left.\beta \gamma\right|_{\Gamma}\right)$ are equivalent, so $H_{\alpha \beta}^{G}(F, \Gamma) \simeq H_{1 \beta \gamma \mid \Gamma}^{G}(F, \Gamma)$. Similarly with $\Gamma$ instead of $F$.

Lemma 2.4. Let $(F, \alpha, \Gamma, \beta)$ be a group-theoretical datum, $H=H_{\alpha \beta}^{G}(F, \Gamma)$, $K=\left\{\bar{g} \in N_{G}(F) / F:[\alpha]=\left[\alpha^{g}\right]\right\}$. Then $G(H)$ fits into an exact sequence

$$
1 \longrightarrow \widehat{F} \hookrightarrow-G(H) \longrightarrow K \longrightarrow 1 \text {. }
$$

Proof: Since $G(H)$ is isomorphic to the group of invertible objects in the tensor category Corep $H \simeq \mathcal{C}(G, 1 ; F, \alpha)$, the claim follows from [GN].

The action and the cocycle in (2.2) are explicitly given in $[\mathbf{G N}]$. In particular, if $\alpha=1$, then $G(H) \simeq \widehat{F} \rtimes N_{G}(F) / F$. 
Lemma 2.5. Let $G^{\prime}$ be a finite group. Let $(F, \alpha, \Gamma, \beta)$ and $\left(F^{\prime}, \alpha^{\prime}, \Gamma^{\prime}, \beta^{\prime}\right)$ be group-theoretical data for $G$ and $G^{\prime}$ respectively. Then $\operatorname{Rep} H_{\alpha \beta}^{G}(F, \Gamma)$ and $\operatorname{Rep} H_{\alpha^{\prime} \beta^{\prime}}^{G^{\prime}}\left(F^{\prime}, \Gamma^{\prime}\right)$ are equivalent as tensor categories if and only if there is an invertible $\operatorname{vec}_{G}-\operatorname{vec}_{G^{\prime}}$-bimodule category $\mathcal{X}$ such that

$$
\mathcal{X} \otimes_{\mathrm{vec}_{G^{\prime}}} \mathcal{M}\left(\Gamma^{\prime}, \beta^{\prime}\right) \simeq \mathcal{M}(\Gamma, \beta)
$$

as $\operatorname{vec}_{G}$-module categories, where $\mathcal{M}\left(\Gamma^{\prime}, \beta^{\prime}\right)$ and $\mathcal{M}(\Gamma, \beta)$ are the module categories associated to the pairs $\left(\Gamma^{\prime}, \beta^{\prime}\right)$ and $(\Gamma, \beta)$.

Proof: Using Rep $H_{\alpha \beta}^{G}(F, \Gamma) \simeq_{\otimes} \mathcal{C}(G, 1 ; \Gamma, \beta)$ and $\operatorname{Rep} H_{\alpha^{\prime} \beta^{\prime}}^{G^{\prime}}\left(F^{\prime}, \Gamma^{\prime}\right) \simeq_{\otimes}$ $\mathcal{C}\left(G^{\prime}, 1 ; \Gamma^{\prime}, \beta^{\prime}\right)$, then [GP, Proposition 5.1] applies.

\section{Group-theoretical Hopf algebras over $G=C_{3} \rtimes C_{6}$}

The group $\mathbb{S}_{3}$ has a Yetter-Drinfeld module $V$ with $\operatorname{dim} \mathfrak{B}(V)=12$ [MS], but there is no non-trivial group-theoretical Hopf algebra over $\mathbb{S}_{3}$. Indeed, the classification of the Hopf algebras of dimension $6=\left|\mathbb{S}_{3}\right|$ is known: $\mathbb{k}_{6}, \mathbb{k S}_{3}$, and $\mathbb{k}^{\mathbb{S}_{3}}$. Hence the only group-theoretical Hopf algebras over $\mathbb{S}_{3}$, up to Morita-equivalence, are $\mathbb{k S}_{3}$ and $\mathbb{k}^{\mathbb{S}_{3}}$.

However the braided vector space $V$ can be realized as a Yetter-Drinfeld module over $G_{m}=C_{3} \rtimes C_{2 m}, m \in \mathbb{N}$, see [GV, Section 4]. Here we deal with the group-theoretical Hopf algebras over $C_{3} \rtimes C_{6}$. Notice that the classification of the semisimple Hopf algebras of dimension 18 is known $[\mathbf{M a}]$.

Let $\xi \in \mathbb{G}_{3}, L=C_{2}=\langle x\rangle, N=C_{3} \times C_{3}=\langle a\rangle \times\langle b\rangle$. The Hopf algebra $A_{18, \xi}$, defined in [Ma, 1.2], is the abelian extension associated to the matched pair $(L, N)$ with $\triangleright: N \times L \rightarrow L$ trivial, $\triangleleft: N \times L \rightarrow N$ given by $a^{i} b^{j} \triangleleft x=a^{i} b^{-j}$ and cocycles $\sigma: L \times L \rightarrow\left(\mathbb{k}^{N}\right)^{\times}$trivial and $\tau: N \times N \rightarrow\left(\mathbb{k}^{L}\right)^{\times}$given by $\tau\left(a^{i} b^{j}, a^{r} b^{s}\right)=\delta_{1}+\xi^{j r} \delta_{x}$. Accordingly, $G=C_{3} \rtimes C_{6}=\langle x, a, b\rangle$. Also, $A_{18, \xi} \simeq A_{18, \eta} \Longleftrightarrow|\xi|=|\eta|[\mathbf{M a}, 1.5]$.

Proposition 3.1. The non-trivial group-theoretical Hopf algebras over $G$ are $A_{18, \xi}$ and $\left(A_{18, \xi}\right)^{*}, \xi \in \mathbb{G}_{3}^{\prime}$.

Proof: Since $|G|=2 \times 3^{2}$, the unique non-trivial subgroup with a nondegenerated 2-cocycle is $N \simeq C_{3} \times C_{3}$. Let $M=\langle x, b\rangle \simeq \mathbb{S}_{3}$. Let $(F, \alpha, \Gamma, \beta)$ be a group-theoretical data for $G$; then $F \cap \Gamma$ is either 1 or $N$.

Case 1: $F \cap \Gamma=1$. Up to conjugation, either $(F, \Gamma)$ or $(\Gamma, F)$ is one of

$$
\begin{array}{llll}
(3.1) & (L, N) & (3.3) & (\langle a\rangle, M) \\
(3.2) & (\langle b\rangle,\langle x, a\rangle) & (3.4) & (\langle a b\rangle, M)
\end{array}
$$

$$
(\langle a b\rangle,\langle x, a\rangle)
$$


If $(F, \Gamma)$ is as in (3.2), (3.3), $F \triangleleft G$ hence $H_{\alpha \beta}^{G}(F, \Gamma)$ is cocommutative, cf. Lemma 2.2(ii). If $(F, \Gamma)$ is as in (3.4), $F \nless G$ and $\Gamma$ is non-abelian, hence $H_{\alpha \beta}^{G}(F, \Gamma)$ is non-trivial. If $(F, \Gamma)$ is as in (3.5), $F, \Gamma \Varangle G$, hence $H_{\alpha \beta}^{G}(F, \Gamma)$ is non-trivial. If $(F, \Gamma)$ is as in (3.1), $F \Varangle G, H^{2}\left(N, \mathbb{k}^{\times}\right) \simeq C_{3}$ and $H^{2}\left(N, \mathbb{R}^{\times}\right)^{\text {ad } G}=1$, then $H_{1 \beta}^{G}(G, N), \beta \neq 1$, is non-trivial. Moreover, these cocycles give rise to isomorphic Hopf algebras.

Case 2: $F \cap \Gamma=N$, so that $(F, \Gamma)$ is either $(N, G)$ - denoted by $(3.6)$ - or else $(G, N)$. Since $H^{2}\left(N, \mathbb{k}^{\times}\right) \simeq C_{3}$ and $H^{2}\left(N, \mathbb{k}^{\times}\right)^{\text {ad } G}=1, H_{1 \beta}^{G}(G, N)$, $\beta \neq 1$, is non-trivial. Further, these cocycles give rise to isomorphic Hopf algebras.

So we have the following possibilities:

\begin{tabular}{|c|c|c|c|c|c|c|}
\hline$\#$ & $F$ & $\alpha$ & $\Gamma$ & $\beta$ & $G(H)$ & $G\left(H^{*}\right)$ \\
\hline$(3.1)$ & $L \simeq C_{2}$ & 1 & $N \simeq C_{3} \times C_{3}$ & $\neq 1$ & $C_{6}$ & $C_{3} \times C_{3}$ \\
\hline$(3.4)$ & $\langle a b\rangle \simeq C_{3}$ & 1 & $M \simeq \mathbb{S}_{3}$ & 1 & $C_{3} \times C_{3}$ & $C_{6}$ \\
\hline$(3.5)$ & $\langle a b\rangle$ & 1 & $\langle x, a\rangle \simeq C_{6}$ & 1 & $C_{3} \times C_{3}$ & $C_{6}$ \\
\hline$(3.6)$ & $N$ & $\neq 1$ & $G$ & 1 & $C_{3} \times C_{3}$ & $C_{6}$ \\
\hline
\end{tabular}

TABLE 2 .

By $[\mathrm{Ma}, 2.3,2.5]$, since $|G(H)|=9,(3.1)^{*} \simeq(3.4) \simeq(3.5) \simeq(3.6) \simeq$ $A_{18, \xi}$; since $|G(H)|=6,(3.1) \simeq(3.4)^{*} \simeq(3.5)^{*} \simeq(3.6)^{*} \simeq\left(A_{18, \xi}\right)^{*}$.

Thus $A_{18, \xi} \simeq(3.6)$ is a twisting of $\mathbb{k} G$.

Theorem 3.2. The Hopf algebras $A_{18, \xi}$ and $\left(A_{18, \xi}\right)^{*}$ have a non-zero Yetter-Drinfeld module $V$ with $\operatorname{dim} \mathfrak{B}(V)<\infty$. By bosonization, we get new Hopf algebras with the dual Chevalley property of dimension 216.

The liftings of $\mathfrak{B}(V) \# \mathbb{k} G$, where $V$ is as above, are classified in $[\mathbf{G V}$, Theorem 6.2]. Indeed, let $\left(x_{j}\right)_{0 \leqslant j \leqslant 2}$ be the basis of $V$ as in loc. cit. Let

$$
\mathcal{S}=\left\{\left(\lambda_{1}, \lambda_{2}\right) \in \mathbb{k}^{2} \text { satisfying }[\mathbf{G V},(29),(33)]\right\} .
$$

For $\left(\lambda_{1}, \lambda_{2}\right) \in \mathcal{S}$, let $H\left(\lambda_{1}, \lambda_{2}\right)$ be $T(V) \# \mathbb{k} G$ modulo the ideal generated by

$$
x_{0}^{2}-\lambda_{1}\left(1-g_{0}^{2}\right) \text { and } x_{0} x_{1}+x_{1} x_{2}+x_{2} x_{0}-\lambda_{2}\left(1-g_{0} g_{1}\right) .
$$

Then

- $H\left(\lambda_{1}, \lambda_{2}\right)$ is a lifting of $\mathfrak{B}(V) \# \mathbb{k} G$,

- any lifting of $\mathfrak{B}(V) \# \mathbb{k} G$ is isomorphic to $H\left(\lambda_{1}, \lambda_{2}\right)$ for some $\left(\lambda_{1}, \lambda_{2}\right) \in \mathcal{S}$

- $H\left(\lambda_{1}, \lambda_{2}\right) \simeq H\left(\lambda_{1}^{\prime}, \lambda_{2}^{\prime}\right)$ iff there exists $\mu \in \mathbb{R}^{\times}$such that $\left(\lambda_{1}, \lambda_{2}\right)=$ $\mu\left(\lambda_{1}^{\prime}, \lambda_{2}^{\prime}\right)$. 
Remark 3.3. The classification of all liftings of $\mathfrak{B}(V) \# A_{18, \xi}$ follows from $\left[\mathbf{G V}\right.$, Theorem 6.2]. Namely, let $J \in \mathbb{k} G \otimes \mathbb{k} G$ such that $A_{18, \xi} \simeq(\mathbb{k} G)^{J}$. Then

- $H\left(\lambda_{1}, \lambda_{2}\right)^{J}$ is a lifting of $\mathfrak{B}(V) \# A_{18, \xi}$, for every $\left(\lambda_{1}, \lambda_{2}\right) \in \mathcal{S}$,

- any lifting of $\mathfrak{B}(V) \# A_{18, \xi}$ is $\simeq$ to $H\left(\lambda_{1}, \lambda_{2}\right)^{J}$ for some $\left(\lambda_{1}, \lambda_{2}\right) \in \mathcal{S}$,

- $H\left(\lambda_{1}, \lambda_{2}\right)^{J} \simeq H\left(\lambda_{1}^{\prime}, \lambda_{2}^{\prime}\right)^{J}$ iff there is $\mu \in \mathbb{k}^{\times}$such that $\left(\lambda_{1}, \lambda_{2}\right)=$ $\mu\left(\lambda_{1}^{\prime}, \lambda_{2}^{\prime}\right)$.

\section{Group-theoretical Hopf algebras over $G=C_{5} \rtimes_{2} C_{20}$}

The group $G=C_{5} \rtimes_{2} C_{4}$ has two Yetter-Drinfeld modules $V_{j}, j=$ 2,3 , with $\operatorname{dim} \mathfrak{B}\left(V_{j}\right)=1280$ [AG1]; $V_{j}$ has braided vector space with rack $Q_{5, j}$ and cocycle -1 , and $V_{2} \simeq V_{3}^{*}$ in $\mathbb{k}_{k} \mathbb{Y}^{k} \mathcal{D}$. But a group-theoretical Hopf algebra over $C_{5} \rtimes_{2} C_{4} \simeq C_{5} \rtimes_{3} C_{4}$ is trivial. Indeed, a non-trivial subgroup of $C_{5} \rtimes_{2} C_{4}$ does not admit a non-degenerated 2-cocycle, (alternatively, there is no triangular Hopf algebra of dimension $20[\mathbf{G e}$, Nat1]). Thus such a group-theoretical Hopf algebra would be an abelian extension, hence trivial.

However the braided vector spaces $V_{j}, j=2$ or 3 , can be realized as Yetter-Drinfeld modules over $C_{5} \rtimes_{2} C_{4 m} \simeq C_{5} \rtimes_{3} C_{4 m}, m \in \mathbb{N}[\mathbf{G V}$, Section 4]. Here we deal with group-theoretical Hopf algebras over $C_{5} \rtimes_{2}$ $\mathrm{C}_{20}$.

Proposition 4.1. The non-trivial group-theoretical Hopf algebras over $G=C_{5} \rtimes_{2} C_{20}$ are given by the group-theoretical data in Table 3.

Question 4.2. Is it true that $4 . a \simeq 4 . d \simeq 4 . f \simeq 4 . h$ ?

\begin{tabular}{|c|c|c|c|c|c|}
\hline$\#$ & $F$ & $\alpha$ & $\Gamma$ & $\beta$ & $G(H)$ \\
\hline $4 . \mathrm{a}$ & $\langle x\rangle \simeq C_{4}$ & 1 & $N \simeq C_{5} \times C_{5}$ & $\neq 1$ & $C_{20}$ \\
\hline $4 . \mathrm{b}$ & $N$ & $\neq 1$ & $\langle x\rangle$ & 1 & $C_{5} \times C_{5}$ \\
\hline $4 . \mathrm{c}$ & $\langle a b\rangle \simeq C_{5}$ & 1 & $\langle b, x\rangle \simeq C_{5} \rtimes C_{4}$ & 1 & $C_{5} \times C_{5}$ \\
\hline $4 . \mathrm{d}$ & $\langle b, x\rangle$ & 1 & $\langle a b\rangle$ & 1 & $C_{20}$ \\
\hline 4.e & $\langle a b\rangle \simeq C_{5}$ & 1 & $\left\langle x^{3} a^{2}\right\rangle \simeq C_{20}$ & 1 & $C_{5} \times C_{5}$ \\
\hline 4.f & $\left\langle x^{3} a^{2}\right\rangle$ & 1 & $\langle a b\rangle$ & 1 & $C_{20}$ \\
\hline $4 . \mathrm{g}$ & $N \simeq C_{5} \times C_{5}$ & $\neq 1$ & $G$ & 1 & $C_{5} \times C_{5}$ \\
\hline $4 . \mathrm{h}$ & $G$ & 1 & $N$ & $\neq 1$ & $C_{20}$ \\
\hline
\end{tabular}

TABLE 3. Group-theoretical data for $C_{5} \rtimes_{2} C_{20}$. 
Proof: Let $G=\langle a, b, x\rangle$, where $|a|=|b|=5,|x|=4$, $a$ is central, and $x b x^{-1}=b^{2}$. Then $N=\langle a, b\rangle \simeq C_{5} \times C_{5}$ is a normal 5-Sylow subgroup. Since $G$ has no subgroup isomorphic to $C_{2} \times C_{2}$, the unique non-trivial subgroup with a non-degenerate 2-cocycle is $N$. Let $(F, \alpha, \Gamma, \beta)$ be a group-theoretical data for $G$; then $F \cap \Gamma$ is either 1 or $N$.

Case 1: $F \cap \Gamma=1$. Up to conjugation, either $(F, \Gamma)$ or $(\Gamma, F)$ is one of

(4.1) $\quad(\langle x\rangle, N)$

$(4.2) \quad\left(\langle b\rangle,\left\langle x^{3} a^{2}\right\rangle\right)$

$(4.3) \quad(\langle a\rangle,\langle b, x\rangle)$

(4.4) $\quad(\langle a b\rangle,\langle b, x\rangle)$
(4.5) $\left(\langle a b\rangle,\left\langle x^{3} a^{2}\right\rangle\right)$.

If $(F, \Gamma)$ is as in (4.1), $F \Varangle G, H^{2}\left(\Gamma, \mathbb{k}^{\times}\right)^{\text {ad } G}=1$, and $H^{2}\left(\Gamma, \mathbb{k}^{\times}\right) \simeq C_{5}$, then $H_{1 \beta}^{G}(F, \Gamma), \beta \neq 1$, is non-trivial. Moreover, these cocycles give rise to isomorphic Hopf algebras, denoted by 4.a. If $(F, \Gamma)$ is as in $(4.2),(4.3)$, $F \triangleleft G$ hence $H_{\alpha \beta}^{G}(F, \Gamma)$ is cocommutative, cf. Lemma 2.2(ii). If $(F, \Gamma)$ is as in (4.4), $F \nless G$ and $\Gamma$ is non-abelian, then $H_{\alpha \beta}^{G}(F, \Gamma)$ is nontrivial, giving 4.c. If $(F, \Gamma)$ is as in $(4.5), F, \Gamma \nless G$ hence $H_{\alpha \beta}^{G}(F, \Gamma)$ is non-trivial, thus 4.e.

Case 2: $F \cap \Gamma=N$. Up to conjugation, either $(F, \Gamma)$ or $(\Gamma, F)$ is $(N, G)$. Since $H^{2}\left(\Gamma, \mathbb{k}^{\times}\right)^{\text {ad } G}=1$ and $H^{2}\left(\Gamma, \mathbb{k}^{\times}\right) \simeq C_{5}, H_{1 \beta}^{G}(F, \Gamma), \beta \neq 1$, is non-trivial. Moreover, these cocycles give isomorphic Hopf algebras, i.e. $4 . g$.

Observe that $4 . \mathrm{g}$ is a twisting of $\mathbb{k} G$.

Theorem 4.3. The Hopf algebras from Table 3 have two dual nonzero Yetter-Drinfeld module $V$ with $\operatorname{dim} \mathfrak{B}(V)=1280$. By bosonization, we get new Hopf algebras with the dual Chevalley property of dimension 128000 .

The liftings of $\mathfrak{B}(V) \# \mathbb{k} G$, where $V$ is as above, are classified in $[\mathbf{G V}$, Theorem 6.4, Theorem 6.5]. Indeed, let $\left(x_{j}\right)_{0 \leqslant j \leqslant 4}$ be the basis of $V$ as in loc. cit. Let

$$
\mathcal{S}=\left\{\left(\lambda_{1}, \lambda_{2}, \lambda_{3}\right) \in \mathbb{k}^{2} \text { satisfying }[\mathbf{G V},(29),(33)]\right\} .
$$

For $\left(\lambda_{1}, \lambda_{2}, \lambda_{3}\right) \in \mathcal{S}$, let $H\left(\lambda_{1}, \lambda_{2}, \lambda_{3}\right)$ be $T(V) \# \mathbb{k} G$ modulo the ideal generated by

$$
\begin{gathered}
x_{0}^{2}-\lambda_{1}\left(1-g_{0}^{2}\right), \quad x_{0} x_{1}+x_{2} x_{0}+x_{3} x_{2}+x_{1} x_{3}-\lambda_{2}\left(1-g_{0} g_{1}\right), \quad \text { and } \\
x_{1} x_{0} x_{1} x_{0}+x_{0} x_{1} x_{0} x_{1}-s_{X}-\lambda_{3}\left(1-g_{0}^{2} g_{1} g_{2}\right),
\end{gathered}
$$


for

$$
\begin{aligned}
s_{X}=\lambda_{2}\left(x_{1} x_{0}+x_{0} x_{1}\right)+ & \lambda_{1} g_{1}^{2}\left(x_{3} x_{0}+x_{2} x_{3}\right) \\
& -\lambda_{1} g_{0}^{2}\left(x_{2} x_{4}+x_{1} x_{2}\right)+\lambda_{2} \lambda_{1} g_{0}^{2}\left(1-g_{1} g_{2}\right) ;
\end{aligned}
$$

or by

$$
\begin{gathered}
x_{0}^{2}-\lambda_{1}\left(1-g_{0}^{2}\right), \quad x_{1} x_{0}+x_{0} x_{2}+x_{2} x_{3}+x_{3} x_{1}-\lambda_{2}\left(1-g_{0} g_{1}\right), \quad \text { and } \\
x_{0} x_{2} x_{3} x_{1}+x_{1} x_{4} x_{3} x_{0}-s_{X}^{\prime}-\lambda_{3}\left(1-g_{0}^{2} g_{1} g_{3}\right),
\end{gathered}
$$

for

$$
\begin{aligned}
s_{X}^{\prime}=\lambda_{2}\left(x_{0} x_{1}+x_{1} x_{0}\right)-\lambda_{1} g_{1}^{2}\left(x_{3} x_{2}+x_{0} x_{3}\right) \\
\quad-\lambda_{1} g_{0}^{2}\left(x_{3} x_{4}+x_{1} x_{3}\right)+\lambda_{1} \lambda_{2}\left(g_{1}^{2}+g_{0}^{2}-2 g_{0}^{2} g_{1} g_{3}\right) .
\end{aligned}
$$

Then

- $H\left(\lambda_{1}, \lambda_{2}, \lambda_{3}\right)$ is a lifting of $\mathfrak{B}(V) \# \mathbb{k} G$,

- any lifting of $\mathfrak{B}(V) \# \mathbb{k} G$ is $\simeq$ to $H\left(\lambda_{1}, \lambda_{2}, \lambda_{3}\right)$ for some $\left(\lambda_{1}, \lambda_{2}, \lambda_{3}\right) \in$ $\mathcal{S}$

- $H\left(\lambda_{1}, \lambda_{2}, \lambda_{3}\right) \simeq H\left(\lambda_{1}^{\prime}, \lambda_{2}^{\prime}, \lambda_{3}^{\prime}\right)$ iff there exists $\mu \in \mathbb{k}^{\times}$such that $\left(\lambda_{1}, \lambda_{2}, \lambda_{3}\right)=\mu\left(\lambda_{1}^{\prime}, \lambda_{2}^{\prime}, \lambda_{3}^{\prime}\right)$.

Remark 4.4. Let $H$ be the Hopf algebra corresponding to 4.g. The classification of all liftings of $\mathfrak{B}(V) \# H$ follows from $[\mathbf{G V}$, Theorem 6.4 , Theorem 6.5]. Namely, let $J \in \mathbb{k} G \otimes \mathbb{k} G$ such that $H \simeq(\mathbb{k} G)^{J}$. Then

- $H\left(\lambda_{1}, \lambda_{2}, \lambda_{3}\right)^{J}$ is a lifting of $\mathfrak{B}(V) \# H$, for every $\left(\lambda_{1}, \lambda_{2}, \lambda_{3}\right) \in \mathcal{S}$,

- any lifting of $\mathfrak{B}(V) \# H$ is $\simeq$ to $H\left(\lambda_{1}, \lambda_{2}, \lambda_{3}\right)^{J}$ for some $\left(\lambda_{1}, \lambda_{2}, \lambda_{3}\right) \in$ $\mathcal{S}$

- $H\left(\lambda_{1}, \lambda_{2}, \lambda_{3}\right)^{J} \simeq H\left(\lambda_{1}^{\prime}, \lambda_{2}^{\prime}, \lambda_{3}^{\prime}\right)^{J}$ iff there is $\mu \in \mathbb{k}^{\times}$such that $\left(\lambda_{1}, \lambda_{2}, \lambda_{3}\right)=\mu\left(\lambda_{1}^{\prime}, \lambda_{2}^{\prime}, \lambda_{3}^{\prime}\right)$.

\section{Group-theoretical Hopf algebras over $\mathbb{S}_{4}$}

The classification of the finite-dimensional pointed Hopf algebras over $\mathbb{S}_{4}$ was completed in $[\mathbf{G G}]$; there are exactly three non-zero YetterDrinfeld modules over $\mathbb{k} \mathbb{S}_{4}$ whose Nichols algebra is finite-dimensional and all admit non-trivial deformations. The underlying rack and cocycle are $\left(\mathcal{O}_{2}^{4},-1\right),\left(\mathcal{O}_{2}^{4}, \chi\right)$, or $\left(\mathcal{O}_{4}^{4},-1\right)$. Here we deal with the grouptheoretical Hopf algebras over $G=\mathbb{S}_{4}$; since Out $\mathbb{S}_{4}=1$, we need to describe all group-theoretical data for $G$ up to conjugacy, cf. Lemma 2.2.

Proposition 5.1. The classification of the non-trivial group-theoretical Hopf algebras over $\mathbb{S}_{4}$ is given by the group-theoretical data in Table 4 . 


\begin{tabular}{|c|c|c|c|c|c|}
\hline$\#$ & $F$ & $\alpha$ & $\Gamma$ & $\beta$ & $G(H)$ \\
\hline 5.a & $\langle(34),(13)(24)\rangle \simeq D_{4}$ & 1 & $\langle(243)\rangle \simeq C_{3}$ & 1 & $C_{2} \times C_{2}$ \\
\hline 5.b & $\langle(243)\rangle \simeq C_{3}$ & 1 & $\langle(34),(13)(24)\rangle \simeq D_{4}$ & 1 & $\mathbb{S}_{3}$ \\
\hline 5.c & $\langle(12),(34)\rangle \simeq C_{2} \times C_{2}$ & $\neq 1$ & $\mathbb{S}_{4}$ & 1 & $D_{4}$ \\
\hline 5.d & $\mathbb{S}_{4}$ & 1 & $\langle(12),(34)\rangle$ & $\neq 1$ & $C_{2}$ \\
\hline
\end{tabular}

TABLE 4. Group-theoretical data for $\mathbb{S}_{4}$.

Proof: Since $\left|\mathbb{S}_{4}\right|=2^{3} \times 3$, every non-trivial subgroup that admits a non-degenerated 2-cocycle is isomorphic to $C_{2} \times C_{2}$. Let $(F, \alpha, \Gamma, \beta)$ be a group-theoretical data for $\mathbb{S}_{4}$; then $F \cap \Gamma$ is either trivial or else $\simeq C_{2} \times C_{2}$.

Case 1: Assume that $F \cap \Gamma=1$, i.e. $(F, \Gamma)$ is an exact exact factorization. Up to conjugation, either $(F, \Gamma)$ or $(\Gamma, F)$ is one of

$$
\begin{aligned}
(\langle(34)\rangle,\langle(13)(24),(243)\rangle) & \simeq\left(C_{2}, \mathbb{A}_{4}\right), \\
(\langle(243)\rangle,\langle(34),(13)(24)\rangle) & \simeq\left(C_{3}, D_{4}\right), \\
(\langle(1324)\rangle,\langle(34),(243)\rangle) & \simeq\left(C_{4}, \mathbb{S}_{3}\right), \\
(\langle(14)(23),(13)(24)\rangle,\langle(34),(243)\rangle) & \simeq\left(C_{2} \times C_{2}, \mathbb{S}_{3}\right) .
\end{aligned}
$$

If $(F, \Gamma)$ is as in $(5.4),\langle(14)(23),(13)(24)\rangle \triangleleft \mathbb{S}_{4}$ hence $H_{\alpha \beta}^{\mathbb{S}_{4}}(F, \Gamma)$ is cocommutative. If $(F, \Gamma)$ is as in the remaining cases, $F$ is not normal in $\mathbb{S}_{4}$, $\mathbb{A}_{4}$ is non-abelian and the others $\Gamma$ are not normal, hence $H_{\alpha \beta}^{\mathbb{S}_{4}}(F, \Gamma)$ is non-trivial, cf. Lemma 2.2(ii). Also $H_{\alpha \beta}^{\mathbb{S}_{4}}(F, \Gamma) \simeq H_{11}^{\mathbb{S}_{4}}(F, \Gamma)$ by Remark 2.3.

Case 2: Assume that $F \cap \Gamma \simeq C_{2} \times C_{2}$. Up to conjugation, either $(F, \Gamma)$ or $(\Gamma, F)$ is one of

$$
\begin{aligned}
(\langle(12),(34)\rangle, G) & \simeq\left(C_{2} \times C_{2}, \mathbb{S}_{4}\right), \\
(\langle(14)(23),(13)(24)\rangle, G) & \simeq\left(C_{2} \times C_{2}, \mathbb{S}_{4}\right), \\
(\langle(14)(23),(34)\rangle,\langle(13)(24),(243)\rangle) & \simeq\left(D_{4}, \mathbb{A}_{4}\right) .
\end{aligned}
$$

If $(F, \Gamma)$ is as in $(5.6), H=H_{\alpha 1}^{\mathbb{S}_{4}}\left(F, \mathbb{S}_{4}\right)$ is cocommutative by Lemma $2.2(\mathrm{ii})$; but if it is as in (5.5), then $H$ (a twist of $\mathbb{k}_{\mathbb{S}_{4}}$ ) is non-trivial since $F \Varangle \mathbb{S}_{4}$.

We next deal with $(F, \Gamma)$ as in (5.7). If $\alpha \in H^{2}\left(D_{4}, \mathbb{k}^{\times}\right) \simeq C_{2} \simeq$ $H^{2}\left(\mathbb{A}_{4}, \mathbb{k}^{\times}\right) \ni \beta$, then $\alpha_{\mid F \cap \Gamma} \cdot \beta_{\mid F \cap \Gamma}{ }^{-1} \neq 1$ iff either $\alpha \neq 1$ and $\beta=1$, 
or vice versa. By Lemma 2.2(ii), $H_{\alpha 1}^{\mathbb{S}_{4}}(F, \Gamma)$ and $H_{1 \beta}^{\mathbb{S}_{4}}(F, \Gamma)$ are nontrivial, since $F$ and $\mathbb{A}_{4}$ are non-abelian. By Remark 2.3, $H_{\alpha 1}^{\mathbb{S}_{4}}(F, \Gamma) \simeq$ $H_{1 \beta}^{\mathbb{S}_{4}}(F, \Gamma)$.

If $(\Gamma, F)$ is as in any of the cases $(5.1), \ldots,(5.7)$ above, then $H_{\beta \alpha}^{\mathbb{S}_{4}}(\Gamma, F)$ is dual to $H=H_{\alpha \beta}^{\mathbb{S}_{4}}(F, \Gamma)$ by Lemma $2.2(\mathrm{iii})$. In conclusion, we have the following non-trivial Hopf algebras (with a slight abuse of notation):

$$
\begin{array}{ll}
(5.1): H_{11}^{\mathbb{S}_{4}}\left(C_{2}, \mathbb{A}_{4}\right), & (5.1)^{*}: H_{11}^{\mathbb{S}_{4}}\left(\mathbb{A}_{4}, C_{2}\right) ; \\
(5.2): H_{11}^{\mathbb{S}_{4}}\left(C_{3}, D_{4}\right), & (5.2)^{*}: H_{11}^{\mathbb{S}_{4}}\left(D_{4}, C_{3}\right) ; \\
(5.3): H_{11}^{\mathbb{S}_{4}}\left(C_{4}, \mathbb{S}_{3}\right), & (5.3)^{*}: H_{11}^{\mathbb{S}_{4}}\left(\mathbb{S}_{3}, C_{4}\right) ; \\
(5.5): H_{\alpha 1}^{\mathbb{S}_{4}}\left(C_{2} \times C_{2}, \mathbb{S}_{4}\right), & (5.5)^{*}: H_{1 \beta}^{\mathbb{S}_{4}}\left(\mathbb{S}_{4}, C_{2} \times C_{2}\right) ; \\
(5.7): H_{1 \beta}^{\mathbb{S}_{4}}\left(D_{4}, \mathbb{A}_{4}\right), & (5.7)^{*}: H_{\alpha 1}^{\mathbb{S}_{4}}\left(\mathbb{A}_{4}, D_{4}\right) .
\end{array}
$$

By Lemma 5.2 below, the Hopf algebras (5.1), (5.2)*, and (5.7) are twist-equivalent, but since $\mathcal{C}\left(\mathbb{S}_{4}, 1 ;\langle(234)\rangle, 1\right)$ admits a unique fiber functor, then all of them are isomorphic - this gives 5.a, with dual 5.b. Similarly, by Lemma 5.2, the Hopf algebras (5.3) and (5.5) are twist-equivalent, hence isomorphic because $\mathbb{k S}_{4}$ admits a unique non-trivial twisting - this gives 5.c, with dual 5.d. The computation of the various $G(H)$ is performed via Lemma 2.4; hence the Hopf algebras in Table 4 are not isomorphic to each other.

Lemma 5.2. (i) $\mathcal{C}\left(\mathbb{S}_{4}, 1 ;\langle(34),(243)\rangle, 1\right) \cong_{\otimes} \mathcal{C}\left(\mathbb{S}_{4}, 1 ; \mathbb{S}_{4}, \alpha\right) \cong_{\otimes} \operatorname{Rep} \mathbb{S}_{4}$, where $\alpha \in H^{2}\left(\mathbb{S}_{4}, \mathbb{k}^{\times}\right)$.

(ii) $\mathcal{C}\left(\mathbb{S}_{4}, 1 ;\langle(234)\rangle, 1\right) \cong_{\otimes} \mathcal{C}\left(\mathbb{S}_{4}, 1 ; \mathbb{A}_{4}, \beta\right)$, where $\beta \in H^{2}\left(\mathbb{A}_{4}, \mathbb{k}^{\times}\right)$.

Proof: Let us recall some results related with invertible bimodules over pointed fusion categories and the tensor product of their module categories:

If $\mathcal{X}$ is an invertible $\operatorname{vec}_{G}$-bimodule category then as right $\operatorname{vec}_{G}$-module category $\mathcal{X} \cong \mathcal{M}(A, \alpha)$, where $A \triangleleft G$ is abelian and $\alpha \in H^{2}\left(A, \mathbb{k}^{\times}\right)^{\text {ad } G}$, cf. [GP, Corollary 7.11]. In the case of $G=\mathbb{S}_{4}$, there are invertible bimodule categories $\mathcal{X}$ such that as right $\operatorname{vec}_{\mathbb{S}_{4}}$-module category $\mathcal{X}=\mathcal{M}(N, \alpha)$, where $N$ is the Klein normal subgroup of $\mathbb{S}_{4}$, cf. [NR, Subsection 8.2].

The rank of $\mathcal{M}(F, \alpha) \bigotimes_{\mathrm{vec}_{S_{4}}} \mathcal{M}(\Gamma, \beta)$ can be calculated as follows: Let $X:=F \backslash G$ and $Y:=G / \Gamma$ the right and left transitive $G$-sets associated. The groups $G$ acts on $X \times Y$ as $g \cdot(x, y)=\left(x g^{-1}, g y\right)$. Let $\left\{\left(x_{i}, y_{i}\right)\right\}_{i \in F \backslash G / \Gamma}$ be a set of representatives of the orbits of $G$ 
in $X \times Y$ (the set of $G$-orbits is in correspondence with the $(F, \Gamma)$-double cosets), then there is a bijective correspondence between simple objects in $\mathcal{M}(F, \alpha) \bigotimes_{\mathrm{vec}_{\mathbb{S}_{4}}} \mathcal{M}(\Gamma, \beta)$ and irreducible representations of $\mathbb{k}_{\alpha_{i}} \operatorname{Stab}_{G}\left(x_{i}, y_{i}\right)$, where $\alpha_{i}$ are certain 2-cocycles associated with $\alpha$ and $\beta$, cf [GP, Theorem 7.15, Corollary 7.16].

(i) By Lemma 2.5 we have to see that there is an invertible vec $_{\mathbb{S}_{4}}$-bimodule category $\mathcal{X}$ such that

$$
\mathcal{X} \otimes_{\operatorname{vec}_{\mathbb{S}_{4}}} \mathcal{M}(\langle(34),(243)\rangle, 1) \cong \mathcal{M}\left(\mathbb{S}_{4}, \alpha\right),
$$

since $\mathcal{C}\left(\mathbb{S}_{4}, 1 ; \mathbb{S}_{4}, \alpha\right) \cong \operatorname{Rep}_{4}$ for all $\alpha \in H^{2}\left(\mathbb{S}_{4}, \mathbb{k}^{\times}\right)$.

Let $\mathcal{X}$ be an invertible bimodule categories such that as right $\operatorname{vec}_{\mathbb{S}_{4}}$ module category $\mathcal{X}=\mathcal{M}(N, \alpha)$, where $N$ is the Klein normal subgroup of $\mathbb{S}_{4}$. The rank of $\mathcal{M}(N, \alpha) \bigotimes_{\text {vec }_{\mathbb{S}_{4}}} \mathcal{M}(\langle(34),(243)\rangle, 1)$ is one, since $N$ and $\langle(34),(243)\rangle$ are an exact factorization of $\mathbb{S}_{4}$.

The module categories $\mathcal{M}\left(\mathbb{S}_{4}, \alpha\right)$ are characterized as the $\operatorname{vec}_{\mathbb{S}_{4}}$-module categories of rank one. So, $\mathcal{X} \bigotimes_{\mathrm{vec}_{\mathbb{S}_{4}}} \mathcal{M}(\langle(34),(243)\rangle, 1) \cong \mathcal{M}\left(\mathbb{S}_{4}, \alpha\right)$.

(ii) Again, let $\mathcal{X}$ be an invertible bimodule categories such that as right $\operatorname{vec}_{\mathbb{S}_{4}}$-module category $\mathcal{X}=\mathcal{M}(N, \alpha)$, where $N$ is the Klein normal subgroup of $\mathbb{S}_{4}$. Then rank $\left.\mathcal{M}(N, \alpha) \bigotimes_{\mathrm{vec}_{\mathbb{S}_{4}}} \mathcal{M}(\langle\langle(234)\rangle)\rangle, 1\right)$ is two, since there are only two $(\langle(234)\rangle, N)$-double cosets and their stabilizers are trivial.

The module categories $\mathcal{M}\left(\mathbb{A}_{4}, \beta\right)$ are characterized as the vec $\mathbb{S}_{4}$-module categories of rank two. So, $\mathcal{X} \otimes_{\text {vec }_{4}} \mathcal{M}(\langle(234)\rangle, \alpha) \cong \mathcal{M}\left(\mathbb{A}_{4}, \beta\right)$.

Then the Hopf algebra associated to $5 . c$ is a twisting of $\mathbb{k} \mathbb{S}_{4}$.

Theorem 5.3. The group-theoretical Hopf algebras in Table 4 have exactly 3 non-zero Yetter-Drinfeld modules whose Nichols algebra is finitedimensional. By bosonization, we get new Hopf algebras with the dual Chevalley property of dimension 13824.

The liftings of $\mathfrak{B}(V) \# \mathbb{k} \mathbb{S}_{4}$, where $V$ is as above, are classified in [GG, Proposition 5.3]. Indeed, for the ql-data [GG, Definition 3.5]

- $\mathcal{Q}_{4}^{-1}[t]=\left(\mathbb{S}_{4}, \mathcal{O}_{2}^{4}, 1, \cdot, \iota,\{0, \Lambda, \Gamma\}\right)$,

- $\mathcal{Q}_{4}^{\chi}[\lambda]=\left(\mathbb{S}_{4}, \mathcal{O}_{2}^{4}, \chi, \cdot, \iota,\{0,0, \lambda\}\right)$, and

- $\mathcal{D}[t]=\left(\left(\mathbb{S}_{4}, \mathcal{O}_{4}^{4}, 1, \cdot, \iota,\{\Lambda, 0, \Gamma\}\right)\right)$,

where $\Lambda, \Gamma, \lambda \in \mathbb{k}, t=(\Lambda, \Gamma)$, let $\mathcal{H}\left(\mathcal{Q}_{4}^{-1}[t]\right), \mathcal{H}\left(\mathcal{Q}_{4}^{\chi}[\lambda]\right)$, and $\mathcal{H}(\mathcal{D}[t])$, respectively, be the algebras presented by generators and relations 
- $\left\{a_{i}, H_{r}: i \in \mathcal{O}_{4}^{2}, r \in \mathbb{S}_{4}\right\}$,

$$
H_{e}=1, \quad H_{r} H_{s}=H_{r s}, \quad r, s \in \mathbb{S}_{4},
$$$$
H_{j} a_{i}=-a_{j i j} H_{j}, \quad i, j \in \mathcal{O}_{2}^{4} \text {, }
$$$$
a_{(12)}^{2}=0 \text {, }
$$$$
a_{(12)} a_{(34)}+a_{(34)} a_{(12)}=\Gamma\left(1-H_{(12)} H_{(34)}\right),
$$$$
a_{(12)} a_{(23)}+a_{(23)} a_{(13)}+a_{(13)} a_{(12)}=\Lambda\left(1-H_{(12)} H_{(23)}\right) \text {; }
$$

- $\left\{a_{i}, H_{r}: i \in \mathcal{O}_{2}^{4}, r \in \mathbb{S}_{4}\right\}$,

$$
\begin{aligned}
& H_{e}=1, \quad H_{r} H_{s}=H_{r s}, \quad r, s \in \mathbb{S}_{4}, \\
& H_{j} a_{i}=\chi_{i}(j) a_{j i j} H_{j}, \quad i, j \in \mathcal{O}_{2}^{4}, \\
& a_{(12)}^{2}=0, \\
& a_{(12)} a_{(34)}-a_{(34)} a_{(12)}=0, \\
& a_{(12)} a_{(23)}-a_{(23)} a_{(13)}-a_{(13)} a_{(12)}=\lambda\left(1-H_{(12)} H_{(23)}\right) ;
\end{aligned}
$$

- $\left\{a_{i}, H_{r}: i \in \mathcal{O}_{4}^{4}, r \in \mathbb{S}_{4}\right\}$,

$$
\begin{aligned}
& H_{e}=1, \quad H_{r} H_{s}=H_{r s}, \quad r, s \in \mathbb{S}_{4}, \\
& H_{j} a_{i}=-a_{j i j} H_{j}, \quad i \in \mathcal{O}_{4}^{4}, j \in \mathcal{O}_{2}^{4}, \\
& a_{(1234)}^{2}=\Gamma\left(1-H_{(13)} H_{(24)}\right), \\
& a_{(1234)} a_{(1432)}+a_{(1432)} a_{(1234)}=0, \\
& a_{(1234)} a_{(1243)}+a_{(1243)} a_{(1423)}+a_{(1423)} a_{(1234)}=\Gamma\left(1-H_{(12)} H_{(13)}\right) .
\end{aligned}
$$

Then

- these algebras are liftings of $\mathfrak{B}(V) \# \mathbb{R} \mathbb{S}_{4}$,

- any lifting is isomorphic to one of these algebras,

- $\mathcal{H}\left(\mathcal{Q}_{4}^{-1}[t]\right) \simeq \mathcal{H}\left(\mathcal{Q}_{4}^{-1}\left[t^{\prime}\right]\right)$ iff $t \neq 0$ and $t=t^{\prime} \in \mathbb{P}_{\mathbb{k}}^{1}$ or if $t=t^{\prime}=$ $(0,0)$, and the same holds for $\mathcal{H}(\mathcal{D}[t]) ; \mathcal{H}\left(\mathcal{Q}_{4}^{\chi}[\lambda]\right) \simeq \mathcal{H}\left(\mathcal{Q}_{4}^{\chi}[1]\right), \forall \lambda \in$ $\mathbb{k}^{\times}$and $\mathcal{H}\left(\mathcal{Q}_{4}^{\chi}[1]\right) \neq \mathcal{H}\left(\mathcal{Q}_{4}^{\chi}[0]\right)$, [GG, Lemma 6.1].

Remark 5.4. Let $H$ be the Hopf algebra corresponding to 5.c. The classification of all liftings of $\mathfrak{B}(V) \# H$ follows from [GG, Proposition 5.3]. Namely, let $J \in \mathbb{k S}_{4} \otimes \mathbb{k S}_{4}$ such that $H \simeq\left(\mathbb{k S}_{4}\right)^{J}$. Then

- $\mathcal{H}\left(\mathcal{Q}_{4}^{-1}[t]\right)^{J}, \mathcal{H}\left(\mathcal{Q}_{4}^{\chi}[\lambda]\right)^{J}$, and $\mathcal{H}(\mathcal{D}[t])^{J}$ are liftings of $\mathfrak{B}(V) \# H$,

- any lifting of $\mathfrak{B}(V) \# H$ is $\simeq$ to $\mathcal{H}\left(\mathcal{Q}_{4}^{-1}[t]\right)^{J}, \mathcal{H}\left(\mathcal{Q}_{4}^{\chi}[\lambda]\right)^{J}$, or $\mathcal{H}(\mathcal{D}[t])^{J}$, 
- $\mathcal{H}\left(\mathcal{Q}_{4}^{-1}[t]\right)^{J} \simeq \mathcal{H}\left(\mathcal{Q}_{4}^{-1}\left[t^{\prime}\right]\right)^{J}$ iff $t \neq 0$ and $t=t^{\prime} \in \mathbb{P}_{\mathbb{k}}^{1}$ or if $t=t^{\prime}=$ $(0,0)$, and the same holds for $\mathcal{H}(\mathcal{D}[t])^{J} ; \mathcal{H}\left(\mathcal{Q}_{4}^{\chi}[\lambda]\right)^{J} \simeq \mathcal{H}\left(\mathcal{Q}_{4}^{\chi}[1]\right)^{J}$, $\forall \lambda \in \mathbb{k}^{\times}$, and $\mathcal{H}\left(\mathcal{Q}_{4}^{\chi}[1]\right)^{J} \not \mathcal{H}\left(\mathcal{Q}_{4}^{\chi}[0]\right)^{J}$.

\section{Group-theoretical Hopf algebras over $\mathbb{S}_{5}$}

The classification of the finite-dimensional Nichols algebras over $\mathbb{S}_{5}$ is unknown; there are two non-zero Yetter-Drinfeld modules over $\mathbb{k}_{\mathbf{S}} \mathbb{S}_{5}$ with finite-dimensional Nichols algebra $[\mathbf{F K}, \mathbf{G r 2}, \mathbf{G G}]$ and one open case $[\mathbf{A F G V}]$. The underlying rack and cocycles are $\left(\mathcal{O}_{2}^{5},-1\right)$ or $\left(\mathcal{O}_{2}^{5}, \chi\right)$. Here we deal with the group-theoretical Hopf algebras over $G=\mathbb{S}_{5}$ (up to conjugacy because Out $G=1$, cf. Lemma 2.2).

Proposition 6.1. The classification of the non-trivial group-theoretical Hopf algebras over $\mathbb{S}_{5}$ is given by the group-theoretical data in Table 5.

\begin{tabular}{|c|c|c|c|c|c|}
\hline$\#$ & $F$ & $\alpha$ & $\Gamma$ & $\beta$ & $G(H)$ \\
\hline $6 . \mathrm{a}$ & $\langle(45)\rangle \simeq C_{2}$ & 1 & $\langle(12345),(345)\rangle \simeq \mathbb{A}_{5}$ & 1 & $C_{2} \times \mathbb{S}_{3}$ \\
\hline $6 . \mathrm{b}$ & $\langle(12345),(345)\rangle$ & 1 & $\langle(45)\rangle$ & 1 & $C_{2}$ \\
\hline $6 . c$ & $\langle(12345)\rangle \simeq C_{5}$ & 1 & $\langle(345),(2435)\rangle \simeq \mathbb{S}_{4}$ & 1 & $C_{5} \rtimes C_{4}$ \\
\hline $6 . \mathrm{d}$ & $\langle(345),(2435)\rangle$ & 1 & $\langle(12345)\rangle$ & 1 & $C_{2}$ \\
\hline $6 . \mathrm{e}$ & $\langle(345),(45)\rangle \simeq \mathbb{S}_{3}$ & 1 & $\begin{array}{l}\langle(12345),(2354)\rangle \\
\quad \simeq C_{5} \rtimes C_{4}\end{array}$ & 1 & $C_{2} \times C_{2}$ \\
\hline $6 . f$ & $\langle(12345),(2354)\rangle$ & 1 & $\langle(345),(45)\rangle$ & 1 & $C_{4}$ \\
\hline $6 . \mathrm{g}$ & $\langle(12)(345)\rangle \simeq C_{6}$ & 1 & $\begin{array}{l}\langle(12345),(2354)\rangle \\
\quad \simeq C_{5} \rtimes C_{4}\end{array}$ & 1 & $D_{6}$ \\
\hline $6 . \mathrm{h}$ & $\langle(12345),(2354)\rangle$ & 1 & $\langle(12)(345)\rangle$ & 1 & $C_{4}$ \\
\hline $6 . \mathrm{i}$ & $\begin{array}{c}\langle(23)(45),(24)(35)\rangle \\
\simeq C_{2} \times C_{2}\end{array}$ & $\neq 1$ & $\mathbb{S}_{5}$ & 1 & $\left(C_{2} \times C_{2}\right) \rtimes_{\nu} \mathbb{S}_{3}$ \\
\hline $6 . \mathrm{j}$ & $\mathbb{S}_{5}$ & 1 & $\langle(23)(45),(24)(35)\rangle$ & $\neq 1$ & $C_{2}$ \\
\hline $6 . \mathrm{k}$ & $\langle(45),(23)\rangle \simeq C_{2} \times C_{2}$ & $\neq 1$ & $\mathbb{S}_{5}$ & 1 & $D_{4}$ \\
\hline 6.1 & $\mathbb{S}_{5}$ & 1 & $\langle(45),(23)\rangle$ & $\neq 1$ & $C_{2}$ \\
\hline $6 . \mathrm{m}$ & $\langle(45),(24)(35)\rangle \simeq D_{4}$ & $\neq 1$ & $\mathbb{A}_{5}$ & 1 & $C_{2} \times C_{2}$ \\
\hline $6 . \mathrm{n}$ & $\mathbb{A}_{5}$ & 1 & $\langle(45),(24)(35)\rangle$ & $\neq 1$ & $C_{2}$ \\
\hline
\end{tabular}

TABLE 5. Group-theoretical data for $\mathbb{S}_{5}$. 
Proof: Since $\left|\mathbb{S}_{5}\right|=2^{3} \times 3 \times 5$, every non-trivial subgroup that admits a non-degenerated 2-cocycle is isomorphic to $C_{2} \times C_{2}$. Let $(F, \alpha, \Gamma, \beta)$ be a group-theoretical data for $\mathbb{S}_{5}$; then $F \cap \Gamma$ is either trivial or else $\simeq C_{2} \times C_{2}$.

Case 1: Assume that $F \cap \Gamma=1$, i.e. $(F, \Gamma)$ is an exact exact factorization. Up to conjugation, $(F, \Gamma)$ is either of 6.a, 6.c, 6.e, or 6.g, or their transposes 6.b, 6.d, 6.f, or 6.h. Since $\mathbb{A}_{5}$ is non-abelian and the others subgroups in this list are not normal, then $H_{\alpha \beta}^{\mathbb{S}_{5}}(F, \Gamma)$ is non-trivial by Lemma 2.2(ii). Also $H_{\alpha \beta}^{\mathbb{S}_{5}}(F, \Gamma) \simeq H_{1}^{\mathbb{S}_{5}}(F, \Gamma)$ by Remark 2.3.

Case 2: Assume that $F \cap \Gamma \simeq C_{2} \times C_{2}$. Up to conjugation, either $(F, \Gamma)$ or $(\Gamma, F)$ is $6 . \mathrm{i}, 6 . \mathrm{k}$, or $6 . \mathrm{m}$. By Lemma 2.2(ii), since in the first two cases $F \Varangle \mathbb{S}_{5}$, then $H_{\alpha 1}^{\mathbb{S}_{5}}\left(F, \mathbb{S}_{5}\right)$ is non-trivial. We next deal with $(F, \Gamma)$ as in 6.m. If $\alpha \in H^{2}\left(D_{4}, \mathbb{k}^{\times}\right) \simeq C_{2} \simeq H^{2}\left(\mathbb{A}_{5}, \mathbb{k}^{\times}\right) \ni \beta$, then $\alpha_{\mid F \cap \Gamma} \cdot \beta_{\mid F \cap \Gamma}{ }^{-1} \neq 1$ iff either $\alpha \neq 1$ and $\beta=1$, or vice versa. By Lemma 2.2(ii), $H_{\alpha 1}^{\mathbb{S}_{5}}\left(F, \mathbb{A}_{5}\right)$ and $H_{1 \beta}^{\mathbb{S}_{5}}\left(F, \mathbb{A}_{5}\right)$ are non-trivial, since $F$ and $\mathbb{A}_{5}$ are non-abelian. By Remak $2.3, H_{\alpha 1}^{\mathbb{S}_{5}}\left(F, \mathbb{A}_{5}\right) \simeq H_{1 \beta}^{\mathbb{S}_{5}}\left(F, \mathbb{A}_{5}\right)$.

If $(\Gamma, F)$ is as in any of the cases 6.a, 6.c, 6.e, 6.g, 6.i, 6.k, and 6.m, then $H_{\beta \alpha}^{\mathbb{S}_{5}}(\Gamma, F)$ is dual to $H=H_{\alpha \beta}^{\mathbb{S}_{5}}(F, \Gamma)$ by Lemma 2.2(iii). The computation of the various $G(H)$ is performed via Lemma 2.4; hence the Hopf algebras in Table 5 are not isomorphic to each other.

Theorem 6.2. The Hopf algebras from Table 5 have two non-zero Yetter-Drinfeld modules $V$ with $\operatorname{dim} \mathfrak{B}(V)<\infty$. By bosonization, we get new Hopf algebras with the dual Chevalley property of dimension 995328000.

\section{Group-theoretical Hopf algebras over $G=\mathbb{A}_{4} \times C_{2}$}

The classification of the finite-dimensional Nichols algebras over $G=$ $\mathbb{A}_{4} \times C_{2}$ is not known, but there is $V \in{ }_{\mathbb{k} G}^{\mathbb{k} G} \mathcal{Y D}$ with $\operatorname{dim} \mathfrak{B}(V)=72[\mathbf{G r} \mathbf{1}]$; the underlying rack is $\mathcal{T}$ and the cocycle is -1 . The group Aut $G$ is isomorphic to $\mathbb{S}_{4}$, via $\varphi: \mathbb{S}_{4} \rightarrow$ Aut $G$ given by $\varphi(a)(b, i)=\left(a b a^{-1}, i\right)$, for all $a \in \mathbb{S}_{4}, b \in \mathbb{A}_{4}, i \in C_{2}=\{1, x\}$. Let $M<G$,

$$
M:=\langle((13)(24), 1),((12)(34), 1),(1, x)\rangle \simeq C_{2} \times C_{2} \times C_{2} .
$$

Proposition 7.1. The non-trivial group-theoretical Hopf algebras over $G=\mathbb{A}_{4} \times C_{2}$ correspond to the group-theoretical data in Table 6 .

Question 7.2. Is it true that $7 . a \simeq 7 . d \simeq 7 . e \simeq 7 . g \simeq 7 . i \simeq 7 . k$ ? 


\begin{tabular}{|c|c|c|c|c|c|}
\hline \# & $F$ & $\alpha$ & $\Gamma$ & $\beta$ & $G(H)$ \\
\hline 7.a & $\begin{array}{c}\langle((12)(34), x)\rangle \\
\simeq C_{2}\end{array}$ & 1 & $\mathbb{A}_{4} \times 1$ & 1 & $C_{2} \times C_{2} \times C_{2}$ \\
\hline 7.b & $\mathbb{A}_{4} \times 1$ & 1 & $\langle((12)(34), x)\rangle$ & 1 & $C_{6}$ \\
\hline 7.c & $\begin{array}{l}\langle((123), 1)\rangle \\
\quad \simeq C_{3}\end{array}$ & 1 & $M$ & $\begin{array}{c}\notin H^{2}\left(F, \mathbb{R}^{\times}\right)^{\text {ad } G} \\
\left.\alpha\right|_{F \cap \Gamma} \neq 1\end{array}$ & $C_{6}$ \\
\hline 7.d & $M$ & $\begin{array}{c}\notin H^{2}\left(F, \mathbb{k}^{\times}\right)^{\operatorname{ad} G} \\
\left.\alpha\right|_{F \cap \Gamma} \neq 1\end{array}$ & $\langle((123), 1)\rangle$ & 1 & $C_{2} \times C_{2} \times C_{2}$ \\
\hline 7.e & $\begin{array}{l}\langle((14)(23), 1), \\
((12)(34), x)\rangle \\
\quad \simeq C_{2} \times C_{2}\end{array}$ & 1 & $\begin{array}{l}\langle((123), x)\rangle \\
\quad \simeq C_{6}\end{array}$ & 1 & $C_{2} \times C_{2} \times C_{2}$ \\
\hline 7.f & $\langle((123), x)\rangle$ & 1 & $\begin{array}{l}\langle((14)(23), 1), \\
((12)(34), x)\rangle\end{array}$ & 1 & $C_{6}$ \\
\hline $7 . \mathrm{g}$ & $\begin{array}{l}\langle((13)(24), 1), \\
((12)(34), 1)\rangle \\
\quad \simeq C_{2} \times C_{2}\end{array}$ & $\neq 1$ & $G$ & 1 & $C_{2} \times C_{2} \times C_{2}$ \\
\hline 7.h & $G$ & 1 & $\begin{array}{l}\langle((13)(24), 1), \\
((12)(34), 1)\rangle\end{array}$ & $\neq 1$ & $C_{6}$ \\
\hline $7 . \mathrm{i}$ & $\begin{array}{l}\langle((13)(24), x), \\
((12)(34), 1)\rangle \\
\simeq C_{2} \times C_{2}\end{array}$ & $\neq 1$ & $G$ & 1 & $C_{2} \times C_{2} \times C_{2}$ \\
\hline $7 . \mathrm{j}$ & $G$ & 1 & $\begin{array}{c}\langle((13)(24), x), \\
((12)(34), 1)\rangle\end{array}$ & $\neq 1$ & $C_{6}$ \\
\hline $7 . \mathrm{k}$ & $M$ & $\begin{array}{c}\notin H^{2}\left(F, \mathbb{k}^{\times}\right)^{\operatorname{ad} G} \\
\left.\alpha\right|_{F \cap \Gamma} \neq 1\end{array}$ & $\mathbb{A}_{4} \times 1$ & 1 & $C_{2} \times C_{2} \times C_{2}$ \\
\hline 7.1 & $\mathbb{A}_{4} \times 1$ & 1 & $M$ & $\begin{array}{c}\notin H^{2}\left(\Gamma, \mathbb{k}^{\times}\right)^{\operatorname{ad} G} \\
\left.\beta\right|_{F \cap \Gamma} \neq 1\end{array}$ & $C_{6}$ \\
\hline
\end{tabular}

TABLE 6. Group-theoretical data for $G=\mathbb{A}_{4} \times C_{2}$.

Proof: Since $|G|=2^{3} \times 3$, every non-trivial subgroup that admits a nondegenerated 2-cocycle is isomorphic to $C_{2} \times C_{2}$. Let $(F, \alpha, \Gamma, \beta)$ be a group-theoretical data for $\mathbb{S}_{5}$; then $F \cap \Gamma$ is either trivial or else $\simeq C_{2} \times C_{2}$. 
Case 1: Assume that $F \cap \Gamma=1$, i.e. $(F, \Gamma)$ is an exact exact factorization. Up to automorphism, the exact factorizations $(F, \Gamma)$ of $\mathbb{A}_{4} \times C_{2}$ are either

$$
\begin{aligned}
\left(1 \times C_{2}, \mathbb{A}_{4} \times 1\right) & \simeq\left(C_{2}, \mathbb{A}_{4}\right), \\
\left(\langle((12)(34), x)\rangle, \mathbb{A}_{4} \times 1\right) & \simeq\left(C_{2}, \mathbb{A}_{4}\right), \\
(\langle((123), 1)\rangle, M) & \simeq\left(C_{3}, C_{2} \times C_{2} \times C_{2}\right),
\end{aligned}
$$

or their transposes. If $(F, \Gamma)$ as in (7.1) or $(7.4)$, then $H_{\alpha \beta}^{G}(F, \Gamma)$ is cocommutative. If $(F, \Gamma)$ is as in $(7.2), F \Varangle G$ and $\Gamma$ is non-abelian, hence $H_{\alpha \beta}^{G}(F, \Gamma)$ is non-trivial. If $(F, \Gamma)$ is as in $(7.5)$, then $H_{\alpha \beta}^{G}(F, \Gamma)$ is non-trivial, since $F, \Gamma \nless G$. We next deal with $(F, \Gamma)$ as in (7.3). There are six elements in $H^{2}\left(M, \mathbb{k}^{\times}\right)-H^{2}\left(M, \mathbb{k}^{\times}\right)^{\text {ad } G}$ and $\left\langle\operatorname{ad}_{a}\right\rangle<$ Aut $G$ where $a \in G-M$, acts transitively on $H^{2}\left(M, \mathbb{k}^{\times}\right)-H^{2}\left(M, \mathbb{k}^{\times}\right)^{\text {ad } G}$, so we get isomorphic Hopf algebras. For such an $\beta, H_{1 \beta}^{G}(F, \Gamma)$ is non-trivial, as $F \nless G$.

Case 2: $F \cap \Gamma \simeq C_{2} \times C_{2}$. Up to automorphism, either $(F, \Gamma)$ or $(\Gamma, F)$ is one of

$$
\begin{aligned}
(\langle((13)(24), 1),((12)(34), 1)\rangle, G) & \simeq\left(C_{2} \times C_{2}, G\right), \\
(\langle(1, x),((12)(34), 1)\rangle, G) & \simeq\left(C_{2} \times C_{2}, G\right), \\
(\langle((13)(24), x),((12)(34), 1)\rangle, G) & \simeq\left(C_{2} \times C_{2}, G\right), \\
\left(M, \mathbb{A}_{4} \times 1\right) & \simeq\left(C_{2} \times C_{2} \times C_{2}, \mathbb{A}_{4}\right) .
\end{aligned}
$$

If $(F, \Gamma)$ is as in $(7.6)$ then $H_{\alpha}^{G}(F, G)$ is cocommutative. If $(F, \Gamma)$ is as in (7.7) or (7.8), $F \Varangle G$, hence $H_{\alpha 1}^{G}(F, G)$ is non-trivial. We next deal with $(F, \Gamma)$ as in (7.9). For $\alpha \in X, H_{\alpha 1}^{G}\left(M, \mathbb{A}_{4} \times 1\right)$ is not cocommutative; and is not commutative, since $\mathbb{A}_{4} \times 1$ is non-abelian. $H_{1 \beta}^{G}\left(M, \mathbb{A}_{4} \times 1\right)$ is cocommutative. In conclusion, we have the non-trivial Hopf algebras described by the group-theoretical data in Table 6 .

Here 7.g and 7.i are twistings of $\mathbb{k} G$.

Theorem 7.3. The Hopf algebras from Table 6 have a non-zero YetterDrinfeld module $V$ with $\operatorname{dim} \mathfrak{B}(V)=72$. By bosonization, we get new Hopf algebras with the dual Chevalley property of dimension 1728. 
The liftings of $\mathfrak{B}(V) \# \mathbb{k} G$, where $V$ is as above, are classified in [GV , Theorem 6.3]. Indeed, let $\left(x_{j}\right)_{0 \leqslant j \leqslant 2}$ be the basis of $V$ as in loc. cit. Let

$$
\mathcal{S}=\left\{\left(\lambda_{1}, \lambda_{2}, \lambda_{3}\right) \in \mathbb{k}^{2} \text { satisfying }[\mathbf{G V},(29),(33)]\right\} .
$$

For $\left(\lambda_{1}, \lambda_{2}, \lambda_{3}\right) \in \mathcal{S}$, let $H\left(\lambda_{1}, \lambda_{2}, \lambda_{3}\right)$ be $T(V) \# \mathbb{k} G$ modulo the ideal generated by

$$
\begin{gathered}
x_{0}^{2}-\lambda_{1}\left(1-g_{0}^{2}\right), \quad x_{0} x_{1}+x_{1} x_{2}+x_{2} x_{0}-\lambda_{2}\left(1-g_{0} g_{1}\right), \quad \text { and } \\
x_{2} x_{1} x_{0} x_{2} x_{1} x_{0}+x_{1} x_{0} x_{2} x_{1} x_{0} x_{2}+x_{0} x_{2} x_{1} x_{0} x_{2} x_{1}-s_{X}-\lambda_{3}\left(1-g_{0}^{3} g_{1}^{3}\right),
\end{gathered}
$$

where

$$
\begin{aligned}
s_{X}= & \lambda_{2}\left(x_{2} x_{1} x_{0} x_{2}+x_{1} x_{0} x_{2} x_{1}+x_{0} x_{2} x_{1} x_{0}\right)-\lambda_{2}^{3}\left(g_{0} g_{1}-g_{0}^{3} g_{1}^{3}\right) \\
& +\lambda_{1}^{2} g_{0}^{2}\left(g_{3}^{2}\left(x_{2} x_{3}+x_{0} x_{2}\right)+g_{1} g_{3}\left(x_{2} x_{1}+x_{1} x_{3}\right)+g_{1}^{2}\left(x_{1} x_{0}+x_{0} x_{3}\right)\right. \\
& -2 \lambda_{1}^{2} g_{0}^{2}\left(x_{0} x_{3}-x_{2} x_{3}-x_{1} x_{2}+x_{1} x_{0}\right) \\
& -2 \lambda_{1}^{2} g_{2}^{2}\left(x_{2} x_{3}-x_{1} x_{3}+x_{0} x_{2}-x_{0} x_{1}\right) \\
& -2 \lambda_{1}^{2} g_{1}^{2}\left(x_{2} x_{1}+x_{1} x_{3}+x_{1} x_{2}-x_{0} x_{3}+x_{0} x_{1}\right) \\
& +\lambda_{2} \lambda_{1}\left(g_{2}^{2} x_{0} x_{3}+g_{1}^{2} x_{2} x_{3}+g_{0}^{2} x_{1} x_{3}\right) \\
& +\lambda_{2}^{2} g_{0} g_{1}\left(x_{2} x_{1}+x_{1} x_{0}+x_{0} x_{2}-\lambda_{1}\right) \\
& -\lambda_{2} \lambda_{1}^{2}\left(3 g_{0}^{3} g_{3}-2 g_{0} g_{1}^{3}-g_{0}^{2} g_{2}-2 g_{0}^{3} g_{1}+g_{2}-g_{1}^{2}+g_{0}^{2}\right) \\
& -\lambda_{2}\left(\lambda_{1}-\lambda_{2}\right)\left(\lambda_{1} g_{0}^{2}\left(g_{3}^{2}+g_{1} g_{3}+g_{1}^{2}+2 g_{0} g_{1}^{3}\right)+x_{2} x_{1}+x_{1} x_{0}+x_{0} x_{2}\right) .
\end{aligned}
$$

Then

- $H\left(\lambda_{1}, \lambda_{2}, \lambda_{3}\right)$ is a lifting of $\mathfrak{B}(V) \# \mathbb{k} G$,

- any lifting of $\mathfrak{B}(V) \# \mathbb{k} G$ is $\simeq$ to $H\left(\lambda_{1}, \lambda_{2}, \lambda_{3}\right)$ for some $\left(\lambda_{1}, \lambda_{2}, \lambda_{3}\right) \in$ $\mathcal{S}$,

- $H\left(\lambda_{1}, \lambda_{2}, \lambda_{3}\right) \simeq H\left(\lambda_{1}^{\prime}, \lambda_{2}^{\prime}, \lambda_{3}^{\prime}\right)$ iff there exists $\mu \in \mathbb{k}^{\times}$such that $\left(\lambda_{1}, \lambda_{2}, \lambda_{3}\right)=\mu\left(\lambda_{1}^{\prime}, \lambda_{2}^{\prime}, \lambda_{3}^{\prime}\right)$.

Remark 7.4. Let $H$ be the Hopf algebra corresponding either to 7.g or to 7.i. The classification of all liftings of $\mathfrak{B}(V) \# H$ follows from $[\mathbf{G V}$, Theorem 6.3]. Namely, let $J \in \mathbb{k} G \otimes \mathbb{k} G$ such that $H \simeq(\mathbb{k} G)^{J}$. Then 
- $H\left(\lambda_{1}, \lambda_{2}, \lambda_{3}\right)^{J}$ is a lifting of $\mathfrak{B}(V) \# H$, for every $\left(\lambda_{1}, \lambda_{2}, \lambda_{3}\right) \in \mathcal{S}$,

- any lifting of $\mathfrak{B}(V) \# H$ is $\simeq$ to $H\left(\lambda_{1}, \lambda_{2}, \lambda_{3}\right)^{J}$ for some $\left(\lambda_{1}, \lambda_{2}, \lambda_{3}\right) \in$ $\mathcal{S}$,

- $H\left(\lambda_{1}, \lambda_{2}, \lambda_{3}\right)^{J} \simeq H\left(\lambda_{1}^{\prime}, \lambda_{2}^{\prime}, \lambda_{3}^{\prime}\right)^{J}$ iff there is $\mu \in \mathbb{k}^{\times}$such that $\left(\lambda_{1}, \lambda_{2}, \lambda_{3}\right)=\mu\left(\lambda_{1}^{\prime}, \lambda_{2}^{\prime}, \lambda_{3}^{\prime}\right)$.

\section{Group-theoretical Hopf algebras over $G=C_{7} \rtimes_{3} C_{6}$}

The classification of the finite-dimensional Nichols algebras over $G$ is not known, but there are $V_{3}, V_{5} \in{ }_{k \in G}^{\mathbb{k} G} \mathcal{Y D}$ with $\operatorname{dim} \mathfrak{B}\left(V_{j}\right)=326592$, $j=3,5$, see $[\mathbf{G r} \mathbf{1}]$. The underlying racks are $\mathrm{Q}_{7, j}, j=3,5$; in both cases, the cocycle is -1 . Note that $C_{7} \rtimes_{3} C_{6} \simeq C_{7} \rtimes_{5} C_{6}$.

There are two non-trivial semisimple Hopf algebras of dimension 42, $\mathcal{A}_{7}(2,3)$ and $\mathcal{A}_{7}(3,2) \simeq \mathcal{A}_{7}(2,3)^{*} ; G\left(\mathcal{A}_{7}(3,2)\right) \simeq G\left(\mathcal{A}_{7}(2,3)\right) \simeq C_{6}$ and as coalgebras, $\mathcal{A}_{7}(2,3) \simeq \mathbb{k} C_{6} \oplus\left(M_{3}(\mathbb{k})^{*}\right)^{4}$, while $\mathcal{A}_{7}(3,2) \simeq \mathbb{k} C_{6} \oplus$ $\left(M_{2}(\mathbb{k})^{*}\right)^{9}$. See [Nat3, Chapter 10].

Proposition 8.1. The non-trivial group-theoretical Hopf algebras over $G$ are $\mathcal{A}_{7}(2,3)$ and $\mathcal{A}_{7}(3,2)$.

Proof: Let $(F, \alpha, \Gamma, \beta)$ be a group-theoretical data for $G$; then $F \cap \Gamma$ is trivial. Up to conjugation, either $(F, \Gamma)$ or $(\Gamma, F)$ is isomorphic to $\left(C_{2}, C_{7} \rtimes C_{3}\right),\left(C_{3}, D_{7}\right)$, or $\left(C_{6}, C_{7}\right)$. By Lemma $2.2(\mathrm{ii}), H_{11}^{G}\left(C_{6}, C_{7}\right)$ is trivial, while $H:=H_{11}^{G}\left(C_{2}, C_{7} \rtimes C_{3}\right)$ and $H^{\prime}:=H_{11}^{G}\left(C_{3}, D_{7}\right)$ are non-trivial.

Now $H^{*}$ fits into $\mathbb{k}^{C_{7} \rtimes C_{3}} \longrightarrow H^{*} \rightarrow \mathbb{k} C_{2}$. Since $\mathbb{k}^{C_{7} \rtimes C_{3}} \simeq \mathbb{k} C_{3} \oplus$ $\left(M_{3}(\mathbb{k})^{*}\right)^{2}$ as coalgebras, $H^{*} \simeq \mathcal{A}_{7}(2,3)$; then $H \simeq \mathcal{A}_{7}(3,2)$. Also, $H^{\prime *}$ fits

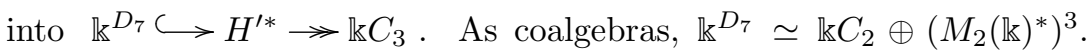
Hence $H^{\prime *} \simeq \mathcal{A}_{7}(3,2)$; therefore $H^{\prime} \simeq \mathcal{A}_{7}(2,3)$.

Theorem 8.2. Each one of $\mathcal{A}_{7}(2,3)$ and $\mathcal{A}_{7}(3,2)$ has two non-zero Yetter-Drinfeld modules $V$ with $\operatorname{dim} \mathfrak{B}(V)=326592$. Thus we get new Hopf algebras with the dual Chevalley property of dimension 13716864.

\section{Group-theoretical Hopf algebras over $D_{n}$}

Let $D_{n}=\left\langle r, s: r^{n}=s^{2}=1\right.$, srs $\left.=r^{-1}\right\rangle$ be the dihedral group of order $2 n$. The classification of the finite-dimensional pointed Hopf algebras over $D_{n}$ is known only when $n=4 t \geqslant 12, t \in \mathbb{N}[\mathbf{F G}]$. Here we deal with the group-theoretical Hopf algebras over $G=D_{n}$ for every $n \geqslant$ 3. We summarize below some well-known facts about $D_{n}$ : 


\begin{tabular}{|c|c|c|}
\hline & $n$ odd & $n$ even \\
\hline$Z\left(D_{n}\right)$ & 1 & $\left\langle r^{n / 2}\right\rangle$ \\
\hline Aut $D_{n}$ & $\left\{\phi_{k, l}:(k, n)=1,0 \leqslant l<n\right\} ;$ & $\phi_{k, l}(r)=r^{k}, \phi_{k, l}(s)=s r^{l}$ \\
\hline Subgroups & $\left\langle r^{d}\right\rangle, d \mid n ;$ & $\left\langle r^{d}, r^{k} s\right\rangle, d \mid n, 0 \leqslant k<d$ \\
\hline $\begin{array}{c}\text { Subgroups } \\
\text { up to Aut } D_{n}\end{array}$ & $\left\langle r^{d}\right\rangle, d \mid n ;$ & $\left\langle r^{d}, s\right\rangle, d \mid n$ \\
\hline $\begin{array}{c}\text { Normal } \\
\text { subgroups }\end{array}$ & $\left\langle r^{d}\right\rangle, d \mid n$ & $\left\langle r^{d}\right\rangle, d \mid n ;\left\langle r^{2}, s\right\rangle ;\left\langle r^{2}, s r\right\rangle$ \\
\hline$\left[D_{n}, D_{n}\right]$ & $\langle r\rangle$ & $\left\langle r^{2}\right\rangle$ \\
\hline$\widehat{D_{n}}$ & $C_{2}$ & $C_{2} \times C_{2}$ \\
\hline$H^{2}\left(D_{n}, \mathbb{C}^{\times}\right)$ & 1 & $C_{2}$ \\
\hline
\end{tabular}

TABLE 7.

In Table 7, when $n$ is even, a representative of the non-trivial class is $f_{\chi} \in Z^{2}\left(D_{n}, \mathbb{k}^{\times}\right), f_{\chi}\left(r^{i} s^{j}, r^{k} s^{l}\right)=\chi\left(r^{k}\right)^{j}, j \in\{0,1\}$, where $\chi:\langle r\rangle \rightarrow \mathbb{k}^{\times}$ is a character of order $n$. Note that $\chi\left(r^{\frac{n}{2}}\right)=-1$.

\subsection{Group-theoretical Hopf algebras over $G=D_{n}, n$ odd.}

Proposition 9.1. Every group-theoretical Hopf algebra over $G$ is trivial.

Proof: Clearly $H^{2}\left(\left\langle r^{d}\right\rangle, \mathbb{k}^{\times}\right)=1=H^{2}\left(D_{n / d}, \mathbb{k}^{\times}\right)$. Let $(F, \alpha, \Gamma, \beta)$ be a group-theoretical data for $D_{n}$; then $F \cap \Gamma=1$. By routine arguments, using that $n$ is odd, and Lemma 2.2, we see that $H_{11}^{D_{n}}(F, \Gamma)$ is trivial.

\subsection{Group-theoretical Hopf algebras over $G=D_{n}, n$ even.}

Proposition 9.2. The classification of the non-trivial group-theoretical Hopf algebras over $G$ is given by the group-theoretical data in Table 8.

Question 9.3. Is it true that 9. $a \simeq 9 . c \simeq 9 . e$ ?

Proof: Let $(F, \alpha, \Gamma, \beta)$ be a group-theoretical datum for $G$. Then $F \cap \Gamma$ is either 1 or else $M=\left\langle r^{n / 2}, s\right\rangle \simeq C_{2} \times C_{2}$, up to equivalence of grouptheoretical data. 


\begin{tabular}{|c|c|c|c|c|c|c|}
\hline$\#$ & $F$ & $\alpha$ & $\Gamma$ & $\beta$ & Condition & $G(H)$ \\
\hline 9. $\mathrm{a}$ & $\begin{array}{c}\left\langle r^{d}, r^{k} s\right\rangle \\
d \mid n \\
0 \leqslant k<d \\
\{d \neq 2 \text { or } \\
\left.\left(d \neq n \text { and } d \neq \frac{n}{2}\right)\right\}\end{array}$ & 1 & $\begin{array}{c}\left\langle r^{e}, s\right\rangle, \\
e \mid n, \\
\{e \neq 2 \text { or } \\
\left.\left(e \neq n \text { and } e \neq \frac{n}{2}\right)\right\}\end{array}$ & 1 & $\begin{array}{l}(d, e)=2, \\
{[d, e]=n,} \\
r^{k} \notin\left\langle r^{2}\right\rangle\end{array}$ & $\begin{array}{c}\text { For } d=2, \\
\left(C_{2} \times C_{2}\right) \rtimes C_{2} \\
\text { if } \frac{n}{2} \text { is even, } \\
C_{2} \times C_{2} \\
\text { if } \frac{n}{2} \text { is odd. } \\
\text { For } d \neq 2, \\
C_{2} \times C_{2} \\
\text { if } \frac{n}{d} \text { is even, } \\
C_{2} \text { if } \frac{n}{d} \text { is odd. }\end{array}$ \\
\hline 9.b & $\begin{array}{c}\left\langle r^{e}, s\right\rangle, \\
e \mid n, \\
\{e \neq 2 \text { or } \\
\left.\left(e \neq n \text { and } e \neq \frac{n}{2}\right)\right\}\end{array}$ & 1 & $\begin{array}{c}\left\langle r^{d}, r^{k} s\right\rangle \\
d \mid n \\
0 \leqslant k<d \\
\{d \neq 2 \text { or } \\
\left.\left(d \neq n \text { and } d \neq \frac{n}{2}\right)\right\}\end{array}$ & 1 & $\begin{array}{l}(d, e)=2, \\
{[d, e]=n,} \\
r^{k} \notin\left\langle r^{2}\right\rangle\end{array}$ & $\begin{array}{l}\text { the same as } \\
\text { before for } e\end{array}$ \\
\hline 9.c & $\left\langle r^{n / 2}, s\right\rangle$ & $\neq 1$ & $D_{n}$ & 1 & $n \neq 4$ & $\begin{array}{c}C_{2} \times C_{2} \\
\text { if } \frac{n}{2} \text { is even, } \\
C_{2} \text { if } \frac{n}{2} \text { is odd. }\end{array}$ \\
\hline 9.d & $D_{n}$ & 1 & $\left\langle r^{n / 2}, s\right\rangle$ & $\neq 1$ & $n \neq 4$ & $C_{2} \times C_{2}$ \\
\hline 9.e & $\begin{array}{c}\left\langle r^{d}, r^{k} s\right\rangle \\
d \mid n, d \neq 1 \\
0 \leqslant k<d \\
\{d \neq 2 \text { or } \\
\left.\left(d \neq n \text { and } d \neq \frac{n}{2}\right)\right\}\end{array}$ & $\neq 1$ & $\begin{array}{c}\left\langle r^{e}, s\right\rangle \\
e \mid n \\
e \neq 1 \\
\{e \neq 2 \text { or } \\
\left.\left(e \neq n \text { and } e \neq \frac{n}{2}\right)\right\}\end{array}$ & 1 & $\begin{array}{l}(d, e)=1, \\
{[d, e]=\frac{n}{2},} \\
r^{k} \in\left\langle r^{d}\right\rangle\end{array}$ & $\begin{array}{c}\text { For } d=2, \\
\left(C_{2} \times C_{2}\right) \rtimes_{\nu} C_{2} \\
\text { if } \frac{n}{2} \text { is even, } \\
C_{2} \times C_{2} \\
\text { if } \frac{n}{2} \text { is odd. } \\
\text { For } d \neq 2, \\
C_{2} \times C_{2} \\
\text { if } \frac{n}{d} \text { is even, } \\
C_{2} \text { if } \frac{n}{d} \text { is odd. }\end{array}$ \\
\hline 9.f & $\begin{array}{c}\left\langle r^{e}, s\right\rangle, \\
e \mid n \\
e \neq 1 \\
\{e \neq 2 \text { or } \\
\left.\left(e \neq n \text { and } e \neq \frac{n}{2}\right)\right\}\end{array}$ & 1 & $\begin{array}{c}\left\langle r^{d}, r^{k} s\right\rangle, \\
d \mid n, d \neq 1, \\
0 \leqslant k<d, \\
\{d \neq 2 \text { or } \\
\left.\left(d \neq n \text { and } d \neq \frac{n}{2}\right)\right\}\end{array}$ & $\neq 1$ & $\begin{array}{l}(d, e)=1, \\
{[d, e]=\frac{n}{2},} \\
r^{k} \in\left\langle r^{d}\right\rangle\end{array}$ & $\begin{array}{c}\text { the same as } \\
\text { 9.a for } e\end{array}$ \\
\hline
\end{tabular}

TABLE 8. Group-theoretical data for $D_{n}, n$ even.

Case 1: $F \cap \Gamma=1$, i.e. $(F, \Gamma)$ is an exact factorization. If $F=\left\langle r^{d}\right\rangle$ and $\Gamma=\left\langle r^{e}, r^{k} s\right\rangle$, then $(d, e)=1$, so $d e=n$, and $H_{11}^{D_{n}}(F, \Gamma)$ is cocommutative. Thus, we may assume that $F=\left\langle r^{d}, r^{k} s\right\rangle$ and $\Gamma=\left\langle r^{e}, s\right\rangle$, up to equivalence of group-theoretical data. We claim that this is an exact factorization iff

$$
(d, e)=2, \quad[d, e]=n, \quad r^{k} \notin\left\langle r^{2}\right\rangle .
$$


First, $F \cap \Gamma=\left(\left\langle r^{d}\right\rangle \cup\left\langle r^{d}\right\rangle r^{k} s\right) \cap\left(\left\langle r^{e}\right\rangle \cup\left\langle r^{e}\right\rangle s\right)=\left\langle r^{[d, e]}\right\rangle \cup\left(\left\langle r^{d}\right\rangle r^{k} s \cap\left\langle r^{e}\right\rangle s\right)$. So $F \cap \Gamma=1$ iff $n \mid[d, e]$ and $r^{k} \notin\left\langle r^{d}\right\rangle\left\langle r^{e}\right\rangle=\left\langle r^{(d, e)}\right\rangle$. If $(F, \Gamma)$ is an exact factorization, then $2 n=|F||\Gamma|=\frac{2 n}{d} \cdot \frac{2 n}{e}$, i.e. $2 n=d e$, and $[d, e]=2 n$ or $n$. But $[d, e]=2 n$ implies $(d, e)=1$ and $r^{k} \in\langle r\rangle$, a contradiction. Thus (9.1) holds. Conversely, if (9.1) holds, then $F \cap \Gamma=1$ and

$$
\begin{aligned}
F \Gamma & =\left\langle r^{d}\right\rangle\left\langle r^{e}\right\rangle \cup\left\langle r^{d}\right\rangle\left\langle r^{e}\right\rangle s \cup\left\langle r^{d}\right\rangle r^{k} s\left\langle r^{e}\right\rangle \cup\left\langle r^{d}\right\rangle r^{k} s\left\langle r^{e}\right\rangle s \\
& =\left\langle r^{2}\right\rangle \cup\left\langle r^{2}\right\rangle s \cup\left\langle r^{2}\right\rangle r^{k} s \cup\left\langle r^{2}\right\rangle r^{k}=D_{n} \text { since } k \text { is odd. }
\end{aligned}
$$

Finally, $F \triangleleft D_{n}$ iff $d=2 ; F$ is abelian iff $d=n$ or $d=\frac{n}{2}$; the same for $\Gamma$. So, we must suppose that $d \neq 2$ or $\left(d \neq n\right.$ and $\left.d \neq \frac{n}{2}\right)$ and $e \neq 2$ or $\left(e \neq n\right.$ and $e \neq \frac{n}{2}$ ). So, $H_{\alpha \beta}^{D_{n}}(F, \Gamma)$ is non-trivial. Now, using Lemma 9.4 $H_{\alpha \beta}^{D_{n}}(F, \Gamma) \simeq H_{11}^{D_{n}}(F, \Gamma)$ this gives 9.a.

Case 2: $F \cap \Gamma=M$. If $(F, \Gamma)=\left(\left\langle r^{n / 2}, s\right\rangle, D_{n}\right)$, then $F \triangleleft D_{n}$ iff $n=4$. By Lemma 2.2(ii), $H_{\alpha 1}^{D_{n}}(F, \Gamma), n \neq 4$, is non-trivial, this gives 9.c.

Now, we may assume that $F=\left\langle r^{d}, r^{k} s\right\rangle$ and $\Gamma=\left\langle r^{e}, s\right\rangle$. We claim that this is a factorization such that $F \cap \Gamma=M$ if and only if

$$
(d, e)=1, \quad[d, e]=\frac{n}{2}, \quad r^{k} \in\left\langle r^{d}\right\rangle .
$$

We have that $F \cap \Gamma=\left\langle r^{n / 2}, s\right\rangle$ iff $[d, e] \equiv \frac{n}{2} \bmod n$ and $r^{k} \in\left\langle r^{\left(\frac{n}{2}, d\right)}\right\rangle$. If $(F, \Gamma)$ is a factorization such that $F \cap \Gamma=M$, then $2 n=\frac{|F| \cdot|\Gamma|}{|F \cap \Gamma|}=$ $\frac{1}{4} \frac{2 n}{d} \frac{2 n}{e}$, i.e. $d e=\frac{n}{2}$, and $[d, e]=\frac{n}{2},(d, e)=1$. Thus (9.2) holds. Conversely, if (9.2) holds, then $F \cap \Gamma=M$ and

$$
\begin{aligned}
F \Gamma & =\left\langle r^{(d, e)}\right\rangle \cup\left\langle r^{(d, e)}\right\rangle s \cup\left\langle r^{(d, e)}\right\rangle r^{k} s \cup\left\langle r^{(d, e)}\right\rangle r^{k} \\
& =\langle r\rangle \cup\langle r\rangle s \cup\langle r\rangle r^{k} s \cup\langle r\rangle r^{k}=D_{n} .
\end{aligned}
$$

If $1 \neq \alpha \in H^{2}\left(D_{n / d}, \mathbb{k}^{\times}\right) \simeq C_{2} \simeq H^{2}\left(D_{n / e}, \mathbb{k}^{\times}\right) \ni \beta \neq 1$, then $\left.\alpha\right|_{F \cap \Gamma} \neq 1,\left.\beta\right|_{F \cap \Gamma} \neq 1$ by Lemma 9.4 below. Further, $H_{\alpha \beta}^{D_{n}}(F, \Gamma), d \neq 2$ or $\left(d \neq n\right.$ and $\left.d \neq \frac{n}{2}\right)$ and $e \neq 2$ or $\left(e \neq n\right.$ and $\left.e \neq \frac{n}{2}\right)$, is non-trivial, this gives 9.e.

Then the Hopf algebra associated to 9.c is a twisting of $\mathbb{k} D_{n}$.

Lemma 9.4. Let $n$ be even. If $F=\left\langle r^{d}, r^{k} s\right\rangle, d \mid n, 0 \leqslant k<d, \frac{n}{d}$ even, then the restriction map Res: $H^{2}\left(D_{n}, \mathbb{C}^{\times}\right) \rightarrow H^{2}\left(F, \mathbb{C}^{\times}\right)$is non-trivial.

Theorem 9.5. Let be $n=4 t, t \geqslant 3$. The Hopf algebras from Table 8 admit families of finite-dimensional Yetter-Drinfeld modules $V$ with $\mathfrak{B}(V)=\Lambda(V)$. Hence we get new finite-dimensional Hopf algebras with the dual Chevalley property. 
The liftings of $\mathfrak{B}(V) \# \mathbb{k} G$, where $V$ is as above, are classified in [FG, Theorem B]. Indeed, the Hopf algebras

- $\mathfrak{B}\left(M_{I}\right) \# \mathbb{k} D_{n}$ with $I=\{(i, k)\} \in \mathcal{I}, k \neq n$,

- $\mathfrak{B}\left(M_{L}\right) \# \mathbb{k} D_{n}$ with $L \in \mathcal{L}$,

- $A_{I}(\lambda, \gamma)$ with $I \in \mathcal{I},|I| \geqslant 1$ or $I=\{(i, n)\}$ and $\gamma \equiv 0$,

- $B_{I, L}(\lambda, \gamma, \theta, \mu)$ with $(I, L) \in \mathcal{K},|I|>0$, and $|L|>0$,

are liftings of $\mathfrak{B}(V) \# \mathbb{k} D_{n}$ and any lifting is isomorphic to one of these algebras. For the definitions see [FG, Definitions 2.6, 2.9, 2.14, 3.9, 3.11]. For the isomorphism classes of these families of Hopf algebras see [FG, Lemmas 3.16, 3.17].

Remark 9.6. Let be $n=4 t, t \geqslant 3$, and $H$ be the Hopf algebra corresponding to 9.c. The classification of all liftings of $\mathfrak{B}(V) \# H$ follows from [FG, Theorem B]. The idea is the same as in Remarks 3.3, 4.4, 5.4, and 7.4 .

\section{References}

[AFGV] N. Andruskiewitsch, F. Fantino, M. Graña, And L. Vendramin, Finite-dimensional pointed Hopf algebras with alternating groups are trivial, Ann. Mat. Pura Appl. (4) 190(2) (2011), 225-245. DOI: 10.1007/s10231-010-0147-0.

[AG1] N. Andruskiewitsch AND M. Graña, From racks to pointed Hopf algebras, Adv. Math. 178(2) (2003), 177-243. DOI: $10.1016 /$ S0001-8708(02)00071-3.

[AG2] N. Andruskiewitsch And M. Graña, Examples of liftings of Nichols algebras over racks. Théories d'homologie, représentations et algèbres de Hopf, AMA Algebra Montp. Announc. (2003), Paper 1, 6 pp.

[AS] N. Andruskiewitsch And H.-J. Schneider, Pointed Hopf algebras, in: "New Directions in Hopf Algebras", Math. Sci. Res. Inst. Publ. 43, Cambridge Univ. Press, Cambridge, 2002, pp. $1-68$.

[AV] N. Andruskiewitsch and C. Vay, Finite dimensional Hopf algebras over the dual group algebra of the symmetric group in three letters, Comm. Algebra 39(12) (2011), 4507-4517. DOI: 10.1080/00927872.2011.616429.

[CDMM] C. Călinescu, S. Dăscălescu, A. Masuoka, and C. MeNINI, Quantum lines over non-cocommutative cosemisimple Hopf algebras, J. Algebra 273(2) (2004), 753-779. DOI: 10.1016/j.jalgebra.2003.08.006. 
[Da] A. A. DAvydov, Galois algebras and monoidal functors between categories of representations of finite groups, J. Algebra 244(1) (2001), 273-301. DOI: 10.1006/jabr.2001.8893.

[EG1] P. ETINGOF AND S. GELAKI, The classification of triangular semisimple and cosemisimple Hopf algebras over an algebraically closed field, Internat. Math. Res. Notices 2000(5) (2000), 223-234. DOI: 10.1155/S1073792800000131.

[EG2] P. Etingof AND S. Gelaki, On families of triangular Hopf algebras, Int. Math. Res. Not. 2002(14) (2002), 757-768. DOI: 10.1155/S1073792802110014.

[ENO] P. Etingof, D. Nikshych, And V. Ostrik, Weakly grouptheoretical and solvable fusion categories, Adv. Math. 226(1) (2011), 176-205. DOI: 10.1016/j.aim.2010.06.009.

[FG] F. Fantino And G. A. García, On pointed Hopf algebras over dihedral groups, Pacific J. Math. 252(1) (2011), 69-91. DOI : $10.2140 /$ pjm.2011.252.69.

[FK] S. Fomin And A. N. KIRILlov, Quadratic algebras, Dunkl elements, and Schubert calculus, in: "Advances in Geometry”, Progr. Math. 172, Birkhäuser Boston, Boston, MA, 1999, pp. $147-182$.

[GP] C. Galindo And J. Y. Plavnik, Tensor functors between Morita duals of fusion categories, Lett. Math. Phys. 107(3) (2017), 553-590. DOI : 10.1007/s11005-016-0914-y.

[GAP] The GAP Group, GAP - Groups, Algorithms, and Programming, Version 4.7.8 (2015). http://www.gap-system.org.

[GG] G. A. García And A. García IGlesias, Finite-dimensional pointed Hopf algebras over $\mathbb{S}_{4}$, Israel J. Math. 183 (2011), 417-444. DOI: 10.1007/s11856-011-0055-z.

[GV] A. García Iglesias and C. VAY, Finite-dimensional pointed or copointed Hopf algebras over affine racks, J. Algebra 397 (2014), 379-406. DOI: 10.1016/j.jalgebra.2013.09.009.

[Ge] S. Gelaki, Quantum groups of dimension $p q^{2}$, Israel J. Math. 102(1) (1997), 227-267. DOI: 10.1007/BF02773801.

[GN] S. GELAKI AND D. NAIDU, Some properties of group-theoretical categories, J. Algebra 322(8) (2009), 2631-2641. DOI : 10. 1016/j.jalgebra.2009.05.047.

[Gr1] M. GraÑA, On Nichols algebras of low dimension, in: "New Trends in Hopf Algebra Theory" (La Falda, 1999), Contemp. Math. 267, Amer. Math. Soc., Providence, RI, 2000, pp. 111-134. DOI: $10.1090 /$ conm/267/04267. 
[Gr2] M. GraÑA, Finite dimensional Nichols algebras of non-diagonal group type, zoo of examples. Web page: http://mate.dm. uba.ar/ matiasg/zoo.html.

[HLV] I. Heckenberger, A. Lochmann, And L. Vendramin, Braided racks, Hurwitz actions and Nichols algebras with many cubic relations, Transform. Groups 17(1) (2012), 157-194. DOI: 10.1007/s00031-012-9176-7.

[Ka] G. I. KAC, Extensions of groups to ring groups, Math. USSR Sbornik 5(3) (1968), 451-474. DOI: 10.1070/SM1968v005n03AB EH003627.

[Ma] A. MasuokA, Some further classification results on semisimple Hopf algebras, Comm. Algebra 24(1) (1996), 307-329. DOI: 10.1080/00927879608825569.

[MS] A. Milinski And H.-J. Schneider, Pointed indecomposable Hopf algebras over Coxeter groups, in: "New Trends in Hopf Algebra Theory" (La Falda, 1999), Contemp. Math. 267, Amer. Math. Soc., Providence, RI, 2000, pp. 215-236. DOI: 10.1090/conm/267/04272.

[Mom] M. Mombelli, Families of finite-dimensional Hopf algebras with the Chevalley property, Algebr. Represent. Theory 16(2) (2013), 421-435. DOI: 10.1007/s10468-011-9313-3.

[Mov] M. V. Movshev, Twisting in group algebras of finite groups, Funct. Anal. Appl. 27(4) (1993), 240-244 (1994); translated from Funktsional. Anal. i Prilozhen. 27(4) (1993), 17-23, 95. DOI : $10.1007 / \mathrm{BF} 01078840$.

[Mü] M. MÜGER, From subfactors to categories and topology. I: Frobenius algebras in and Morita equivalence of tensor categories, J. Pure Appl. Algebra 180(1-2) (2003), 81-157. DOI: 10.1016/S0022-4049(02)00247-5.

[Na] D. NAIDU, Categorical Morita equivalence for group-theoretical categories, Comm. Algebra 35(11) (2007), 3544-3565. DOI: 10.1080/00927870701511996.

[Nat1] S. NAtale, On semisimple Hopf algebras of dimension $p q^{2}, J$. Algebra 221(1) (1999), 242-278. DOI: 10.1006/jabr.1999.7968.

[Nat2] S. NATALE, On group theoretical Hopf algebras and exact factorizations of finite groups, J. Algebra 270(1) (2003), 199-211. DOI: $10.1016 /$ S0021-8693(03)00464-2.

[Nat3] S. NATALE, Semisolvability of semisimple Hopf algebras of low dimension, Mem. Amer. Math. Soc. 186(874) (2007), 123 pp. DOI : $110.1090 / \mathrm{memo} / 0874$. 
[NR] D. Nikshych And B. Riepel, Categorical Lagrangian Grassmannians and Brauer-Picard groups of pointed fusion categories, J. Algebra 411 (2014), 191-214. DOI: 10.1016/j. jalgebra.2014.04.013.

[Os] V. Ostrik, Module categories over the Drinfeld double of a finite group, Int. Math. Res. Not. 2003(27) (2003), 1507-1520. DOI: $10.1155 /$ S1073792803205079.

[Sc1] P. Schauenburg, Hopf bi-Galois extensions, Comm. Algebra 24(12) (1996), 3797-3825. DOI: 10.1080/00927879608825788.

[Sc2] P. Schauenburg, The monoidal center construction and bimodules, J. Pure Appl. Algebra 158(2-3) (2001), 325-346. DOI: $10.1016 /$ S0022-4049(00) 00040-2.

[Sc3] P. SchauenburG, Hopf bimodules, coquasibialgebras, and an exact sequence of Kac, Adv. Math. 165(2) (2002), 194-263. DOI: 10.1006/aima.2001.2016.

[Ta] M. TAKEUCHI, Matched pairs of groups and bismash products of Hopf algebras, Comm. Algebra 9(8) (1981), 841-882. DOI: 10.1080/00927878108822621.

[Ya] S. YamaGami, Group symmetry in tensor categories and duality for orbifolds, J. Pure Appl. Algebra 167(1) (2002), 83-128. DOI: $10.1016 /$ S0022-4049(01)00028-7.

Nicolás Andruskiewitsch and Monique Müller:

Facultad de Matemática, Astronomía y Física

Universidad Nacional de Córdoba

CIEM - CONICET

Medina Allende s/n

(5000) Ciudad Universitaria

Córdoba

Argentina

E-mail address: andrus@famaf .unc.edu.ar

E-mail address: mmuller@famaf.unc.edu.ar

César Galindo:

Department of mathematics

Universidad de los Andes

Carrera 1 No. 18A -12

Bogotá

Colombia

E-mail address: cn.galindo1116@uniandes.edu.co

Primera versió rebuda el 7 de setembre de 2015, darrera versió rebuda el 10 d'octubre de 2016. 